\title{
Low-lying baryon spectrum with two dynamical twisted mass fermions
}

\author{
C. Alexandrou, ${ }^{1,2}$ R. Baron, ${ }^{3}$ J. Carbonell, ${ }^{4}$ V. Drach, ${ }^{4}$ P. Guichon, ${ }^{3}$ K. Jansen, ${ }^{5}$ T. Korzec, ${ }^{1}$ and O. Pène ${ }^{6}$ \\ ${ }^{1}$ Department of Physics, University of Cyprus, P.O. Box 20537, 1678 Nicosia, Cyprus \\ ${ }^{2}$ Computation-based Science and Technology Research Center, Cyprus Institute, 20 Kavafi Strasse, Nicosia 2121, Cyprus \\ ${ }^{3}$ CEA-Saclay, IRFU/Service de Physique Nucléaire, 91191 Gif-sur-Yvette, France \\ ${ }^{4}$ Laboratoire de Physique Subatomique et Cosmologie, UJF/CNRS/IN2P3, 53 avenue des Martyrs, 38026 Grenoble, France \\ ${ }^{5}$ NIC, DESY, Platanenallee 6, D-15738 Zeuthen, Germany \\ ${ }^{6}$ Laboratoire de Physique Théorique (Bâtiment 210), Université de Paris XI, CNRS-UMR8627, Centre d'Orsay, \\ 91405 Orsay-Cedex, France
}

(Received 20 October 2009; published 16 December 2009)

\begin{abstract}
The masses of the low-lying baryons are evaluated using two degenerate flavors of twisted mass sea quarks corresponding to pseudoscalar masses in the range of about 270-500 MeV. The strange valence quark mass is tuned to reproduce the mass of the kaon in the physical limit. The tree-level Symanzik improved gauge action is employed. We use lattices of spatial size 2.1 and $2.7 \mathrm{fm}$ at two values of the lattice spacing with $r_{0} / a=5.22(2)$ and $r_{0} / a=6.61(3)$. We check for both finite volume and cutoff effects on the baryon masses. We performed a detailed study of the chiral extrapolation of the octet and decuplet masses using SU(2) $\chi \mathrm{PT}$. The lattice spacings determined using the nucleon mass at the physical point are consistent with the values extracted using the pion decay constant. We examine the issue of isospin symmetry breaking for the octet and decuplet baryons and its dependence on the lattice spacing. We show that in the continuum limit isospin breaking is consistent with zero, as expected. The baryon masses that we find after taking the continuum limit and extrapolating to the physical limit are in good agreement with experiment.
\end{abstract}

DOI: 10.1103/PhysRevD.80.114503

PACS numbers: 11.15.Ha, 12.38.-t, 12.38.Aw, 12.38.Gc

\section{INTRODUCTION}

In the last couple of years an intense and successful effort in extending unquenched lattice calculations toward realistic values of quark masses, small lattice spacings, and large volumes has been undertaken using a variety of algorithmic techniques and lattice actions. A review of the salient features of the various discretization schemes currently employed can be found in Ref. [1]. Of particular relevance to the current work are the calculations of the low-lying baryon spectrum using two degenerate flavors $\left(N_{f}=2\right)$ of light dynamical quarks. Such studies have been carried out by the MILC Collaboration [2,3] using Kogut-Susskind fermions and by the European Twisted Mass Collaboration (ETMC) [4] for the nucleon $(N)$ and $\Delta$ baryons using twisted mass fermions. There are also baryon mass calculations using two degenerate flavors of light quarks and a strange quark with the mass tuned to its physical value $\left(N_{f}=2+1\right)$ mainly using clover improved Wilson fermions with different levels of smearing, such as the calculation of the nucleon mass by the QCDSFUKQCD Collaboration [5], and the evaluation of the octet and decuplet spectrum by the PACS-CS [6] and BMW [7] Collaborations. The LHP Collaboration computed the octet and decuplet spectrum using a hybrid action with domain wall valence fermions on asqtad improved staggered sea quarks [8]. Preliminary results on the nucleon mass are also computed using $N_{f}=2+1$ domain wall fermions by the RBC-UKQCD Collaboration $[9,10]$.

In this work we study the low-lying spectrum of the baryon octet and decuplet with twisted mass fermions at maximal twist. The light quarks are dynamical degrees of freedom while in the strange sector we use an OsterwalderSeiler valence quark, following the approach employed in the study of the pseudoscalar meson decay constants $[11,12]$. The bare strange valence quark mass is taken to be the same as the one determined in the meson studies tuned by requiring that the mass of the kaon at the physical point matches its physical value. Using the ETMC $N_{f}=2$ configurations $[13,14]$ we calculate the baryon spectrum for pion masses in the range of $270-500 \mathrm{MeV}$ and at two values of the lattice spacing corresponding to $\beta=3.9$ and $\beta=4.05$ with $r_{0} / a=5.22(2)$ and $r_{0} / a=6.61(3)$, respectively, where $r_{0}$ is determined from the force between two static quarks. Results are also obtained at a third $\beta$ value, namely, $\beta=3.8$, which corresponds to $r_{0} / a=$ 4.46(3). The latter results are not taken into account in the final analysis due to large autocorrelation effects observed in the Monte Carlo history for quantities like the PCAC mass and the plaquette at small sea quark masses. Data at $\beta=3.8$ are only used as a consistency check of the continuum extrapolation. For the nucleon mass we also performed the calculation at an even finer value of the lattice spacing corresponding to $r_{0} / a=8.31(5)$ and $\beta=4.2$ to 
ensure that indeed the continuum extrapolation using a weighted average with results at $\beta=3.9$ and $\beta=4.05$ is valid. We find that the baryon masses considered here show a very weak dependence on the lattice spacing and are fully compatible with an $\mathcal{O}\left(a^{2}\right)$ behavior with an almost vanishing coefficient of the $a^{2}$ term. This justifies neglecting the $\mathcal{O}\left(a^{2}\right)$ term in extrapolating results to the continuum limit.

For a fixed value of the lattice spacing we have used up to five different light quark masses and two different volumes. The corresponding $m_{\pi} L$ values are in the range 3.3-7.4, where $L$ is the spatial extent of the lattice. Using these various values of the lattice spacing, quark masses and volumes allows us to estimate the volume corrections and perform a continuum and chiral extrapolation. The good precision of our results on the baryon masses allows us to perform a study of chiral extrapolations to the physical point. This study shows that one of our main uncertainties in predicting the mass at the physical point is caused by the chiral extrapolations. Another source of systematic error is the partially quenched approximation that we have used.

An important issue is the restoration of the explicitly broken isospin symmetry in the continuum limit. At finite lattice spacing, baryon masses display $\mathcal{O}\left(a^{2}\right)$ isospin breaking effects. There are, however, theoretical arguments [15] and numerical evidences [1,16] that these isospin breaking effects are particularly pronounced for the neutral pseudoscalar mass, whereas for other quantities studied so far by ETMC they are compatible with zero. In this paper we will demonstrate that also in the baryon sector these isospin breaking effects are in general small or even compatible with zero. For a preliminary account of these results see Ref. [17].

The paper is organized as follows: The details of our lattice setup, namely, those concerning the twisted mass action, the parameters of the simulations, and the interpolating fields used, are given in Sec. II. Section III contains the numerical results of the baryon masses computed for different lattice volumes, lattice spacings, and bare quark masses as well as the Gell-Mann-Okubo (GMO) relations that are supposed to be fulfilled in the exact SU(3) limit. Lattice artifacts, including finite volume and discretization errors, are discussed in Sec. IV, with special emphasis on the $\mathcal{O}\left(a^{2}\right)$ isospin breaking effects inherent in the twisted mass formulation of lattice QCD. The chiral extrapolations are analyzed in Sec. V. Section VI presents a comparison of our results with those obtained by other collaborations that use dynamical fermion. Conclusions are given in Sec. VII.

\section{LATTICE SETUP}

\section{A. The lattice action}

For the gauge fields we use the tree-level Symanzik improved gauge action [18], which includes besides the plaquette term $U_{x, \mu, \nu}^{1 \times 1}$, also rectangular $(1 \times 2)$ Wilson loops $U_{x, \mu, \nu}^{1 \times 2}$

$$
\begin{aligned}
S_{g}= & \frac{\beta}{3} \sum_{x}\left(b_{0} \sum_{\substack{\mu, \nu=1 \\
1 \leq \mu<\nu}}^{4}\left\{1-\operatorname{Re} \operatorname{Tr}\left(U_{x, \mu, \nu}^{1 \times 1}\right)\right\}\right. \\
& \left.+b_{1} \sum_{\substack{\mu, \nu=1 \\
\mu \neq \nu}}^{4}\left\{1-\operatorname{Re} \operatorname{Tr}\left(U_{x, \mu, \nu}^{1 \times 2}\right)\right\}\right),
\end{aligned}
$$

with $b_{1}=-1 / 12$ and the (proper) normalization condition $b_{0}=1-8 b_{1}$. Note that for $b_{1}=0$ this action reduces to the usual Wilson plaquette gauge action.

The fermionic action for two degenerate flavors of quarks in twisted mass QCD is given by

$$
S_{F}=a^{4} \sum_{x} \bar{\chi}(x)\left(D_{W}[U]+m_{0}+i \mu \gamma_{5} \tau^{3}\right) \chi(x),
$$

with $\tau^{3}$ the Pauli matrix acting in the isospin space, $\mu$ the bare twisted mass, and $D_{W}$ the massless Wilson-Dirac operator given by

$$
D_{W}[U]=\frac{1}{2} \gamma_{\mu}\left(\nabla_{\mu}+\nabla_{\mu}^{*}\right)-\frac{a r}{2} \nabla_{\mu} \nabla_{\mu}^{*}
$$

where

$$
\begin{aligned}
& \nabla_{\mu} \psi(x)=\frac{1}{a}\left[U_{\mu}(x) \psi(x+a \hat{\mu})-\psi(x)\right], \quad \text { and } \\
& \nabla_{\mu}^{*} \psi(x)=-\frac{1}{a}\left[U_{\mu}^{\dagger}(x-a \hat{\mu}) \psi(x-a \hat{\mu})-\psi(x)\right] .
\end{aligned}
$$

Maximally twisted Wilson quarks are obtained by setting the untwisted quark mass $m_{0}$ to its critical value $m_{\mathrm{cr}}$, while the twisted quark mass parameter $\mu$ is kept nonvanishing in order to be away from the chiral limit. In Eq. (2) the quark fields $\chi$ are in the so-called "twisted" basis. The "physical" basis at maximal twist is obtained by the simple transformation

$$
\begin{aligned}
& \psi(x)=\exp \left(\frac{i \pi}{4} \gamma_{5} \tau^{3}\right) \chi(x), \\
& \bar{\psi}(x)=\bar{\chi}(x) \exp \left(\frac{i \pi}{4} \gamma_{5} \tau^{3}\right) .
\end{aligned}
$$

In terms of the physical fields the action is given by

$$
\begin{aligned}
S_{F}^{\psi}= & a^{4} \sum_{x} \bar{\psi}(x)\left(\frac{1}{2} \gamma_{\mu}\left[\nabla_{\mu}+\nabla_{\mu}^{*}\right]\right. \\
& \left.+i \gamma_{5} \tau^{3}\left(-\frac{a r}{2} \nabla_{\mu} \nabla_{\mu}^{*}+m_{\mathrm{cr}}\right)+\mu\right) \psi(x) .
\end{aligned}
$$

In this paper, unless otherwise stated, the quark fields will be understood as physical fields, $\psi$, in particular, when we define the baryonic interpolating fields.

A crucial advantage of the twisted mass formulation is the fact that, by tuning the bare untwisted quark mass $m_{0}$ to its critical value $m_{\mathrm{cr}}$, physical observables are automatically $\mathcal{O}(a)$ improved. In practice, we implement maximal twist of Wilson quarks by tuning to zero the bare untwisted 
current quark mass, commonly called PCAC mass, $m_{\mathrm{PCAC}}$, which is proportional to $m_{0}-m_{\mathrm{cr}}$ up to $\mathcal{O}(a)$ corrections. As detailed in Ref. [19], $m_{\mathrm{PCAC}}$ is conveniently evaluated through

$$
m_{\mathrm{PCAC}}=\lim _{t / a \gg 1} \frac{\sum_{\mathbf{x}}\left\langle\partial_{4} \tilde{A}_{4}^{b}(\mathbf{x}, t) \tilde{P}^{b}(0)\right\rangle}{2 \sum_{\mathbf{x}}\left\langle\tilde{P}^{b}(\mathbf{x}, t) \tilde{P}^{b}(0)\right\rangle}, \quad b=1,2,
$$

where $\tilde{A}_{\mu}^{b}=\bar{\chi} \gamma_{\mu} \gamma_{5} \frac{\tau^{b}}{2} \chi$ is the axial vector current and $\tilde{P}^{b}=\bar{\chi} \gamma_{5} \frac{\tau^{b}}{2} \chi$ is the pseudoscalar density in the twisted basis. The large $t / a$ limit is required in order to isolate the contribution of the lowest-lying charged pseudoscalar meson state in the correlators of Eq. (7). This way of determining $m_{\text {PCAC }}$ is equivalent to imposing on the lattice the validity of the axial Ward identity

$$
\partial_{\mu} \tilde{A}_{\mu}^{b}=2 m_{\mathrm{PCAC}} \tilde{P}^{b}, \quad b=1,2
$$

between the vacuum and the charged one-pion zero threemomentum state.

The value of $m_{\mathrm{cr}}$ is determined at each $\beta$ value at the lowest twisted mass used in our simulations, a procedure that preserves $\mathcal{O}(a)$ improvement and keeps $\mathcal{O}\left(a^{2}\right)$ small $[14,20]$. The twisted mass fermionic action breaks parity and isospin at nonvanishing lattice spacing, as it is apparent from the form of the Wilson term in Eq. (6). In particular, the isospin breaking in physical observables is a cutoff effect of $\mathcal{O}\left(a^{2}\right)$ [21].

To simulate the strange quark in the valence sector several choices are possible. We consider a quenched Osterwalder-Seiler fermion [22] with the following action in the twisted basis:

$$
S_{s}=a^{4} \sum_{x} \bar{\chi}_{s}(x)\left(D_{W}[U]+m_{0}+i \mu_{s} \gamma_{5}\right) \chi_{s}(x) \text {. }
$$

This is naturally realized in the twisted mass approach by introducing an additional doublet of strange quark and keeping only the positive diagonal component of $\tau_{3}$. The $m_{0}$ value is taken to be equal to the critical mass determined in the light sector, thus guaranteeing the $O(a)$ improvement in any observable. The reader interested in the advantage of this mixed action in the mesonic sector is referred to Refs. [11,12,23-25].

\section{B. Simulation details}

The input parameters of the calculation, namely, $\beta, L / a$, and $a \mu$ are summarized in Table I. The corresponding lattice spacing $a$ and the pion mass values, spanning a mass range from 270 to $500 \mathrm{MeV}$, are taken from Ref. [26]. At $m_{\pi} \approx 300 \mathrm{MeV}$ we have simulations for lattices of spatial size $L=2.1 \mathrm{fm}$ and $L=2.7 \mathrm{fm}$ at $\beta=$ 3.9 allowing us to investigate finite volume effects. Finite lattice spacing effects are investigated using two sets of results at $\beta=3.9$ and $\beta=4.05$. The set at $\beta=3.8$ is used only as a cross-check and to estimate cutoff errors. These sets of gauge ensembles allow us to estimate the systematic errors in order to extract reliable results for the baryon spectrum.

\section{Tuning of the bare strange quark mass}

In a previous paper from the ETM Collaboration [11], pseudoscalar meson masses have been computed for different values of the sea and valence quark masses for the $\beta=$

\begin{tabular}{|c|c|c|c|c|c|c|}
\hline \multicolumn{7}{|c|}{$\beta=4.05, a=0.0666(6)$ fm from $f_{\pi}[26], r_{0} / a=6.61(3)$} \\
\hline \multirow[t]{4}{*}{$32^{3} \times 64, L=2.13 \mathrm{fm}$} & $a \mu$ & 0.0030 & 0.0060 & 0.0080 & 0.012 & \\
\hline & No. of confs. & 269 & 253 & 409 & 182 & \\
\hline & $m_{\pi}(\mathrm{GeV})$ & $0.3070(18)$ & $0.4236(18)$ & $0.4884(15)$ & $0.6881(18)$ & \\
\hline & $m_{\pi} L$ & 3.31 & 4.57 & 5.27 & 7.43 & \\
\hline \multirow{5}{*}{$24^{3} \times 48, L=2.05 \mathrm{fm}$} & $\beta=3$ & $=0.0855(6)$ & $\operatorname{rom} f_{\pi}$ [26], & $=5.22(2)$ & & \\
\hline & $a \mu$ & 0.0030 & 0.0040 & 0.0064 & 0.0085 & 0.010 \\
\hline & No. of confs. & $\ldots$ & 782 & 545 & 348 & 477 \\
\hline & $m_{\pi}(\mathrm{GeV})$ & $\ldots$ & $0.3131(16)$ & $0.3903(9)$ & $0.4470(12)$ & $0.4839(12)$ \\
\hline & $m_{\pi} L$ & & 3.25 & 4.05 & 4.63 & 5.03 \\
\hline \multirow[t]{4}{*}{$32^{3} \times 64, L=2.74 \mathrm{fm}$} & $a \mu$ & 0.0030 & 0.0040 & & & \\
\hline & No. of confs. & 659 & 232 & & & \\
\hline & $m_{\pi}(\mathrm{GeV})$ & $0.2696(9)$ & $0.3082(6)$ & & & \\
\hline & $m_{\pi} L$ & 3.74 & 4.28 & & & \\
\hline \multirow{5}{*}{$24^{3} \times 48, L=2.39 \mathrm{fm}$} & & $3.8, a=0.0$ & $\mathrm{fm}, r_{0} / a=$ & & & \\
\hline & $a \mu$ & 0.0060 & 0.0080 & 0.0110 & 0.0165 & \\
\hline & No. of confs, & 215 & 302 & 248 & 244 & \\
\hline & $m_{\pi}(\mathrm{GeV})$ & $0.3667(17)$ & $0.4128(16)$ & $0.4799(9)$ & $0.5855(10)$ & \\
\hline & $m_{\pi} L$ & 4.44 & 5.00 & 5.81 & 7.09 & \\
\hline
\end{tabular}
3.9 gauge configurations. Using the experimental value of

TABLE I. Input parameters $(\beta, L, \mu)$ of our lattice calculation and corresponding lattice spacing $(a)$ and pion mass $\left(m_{\pi}\right)$. 
TABLE II. Bare strange quark mass used in the valence sector for different $\beta$ values.

\begin{tabular}{cccc}
\hline \hline & $\beta=3.8$ & $\beta=3.9$ & $\beta=4.05$ \\
\hline$a \mu_{s}$ & $0.0208(15)(48)$ & $0.0217(22)$ & $0.0166(18)(29)$ \\
\hline \hline
\end{tabular}

the mass ratio of the kaon to the pion, $m_{K} / m_{\pi}$, the bare strange quark mass can be set. We use the value of $a \mu_{s}=$ $0.0217(22)$ at $\beta=3.9$ taken from Table 2 of Ref. [11]. In a more recent study of the pseudoscalar decay constant of kaons and D mesons [12], the computation was extended to $\beta=3.8$ and $\beta=4.05$. However, this is still a preliminary analysis and an ongoing analysis for the accurate extraction of quark masses is still in progress. One can obtain an estimate of the bare strange quark mass at a given value of $\beta$ by taking the results at $\beta=3.9$ as a reference and using the scaling relation [27]:

$$
a \mu_{s}(\beta)=\frac{Z_{p}(\beta)}{Z_{p}(\beta=3.9)} \frac{a(\beta)}{a(\beta=3.9)} a \mu_{s}(\beta=3.9) .
$$

The values we use for $\beta=3.8$ and $\beta=4.05$ given in Table II are obtained by applying Eq. (10). We use the value of the renormalization constant $Z_{p}(\beta)$ found in the preliminary analysis of Ref. [28] within the RI'-MOM, a nonperturbative renormalization scheme [29]. This value is in agreement with a complementary analysis given in Ref. [30].

\section{Interpolating fields}

The low-lying baryons belonging to the octet and decuplet representations of SU(3) are given in Figs. 1 and 2, respectively. They are classified by giving the isospin, $I$, the third component of the isospin, $I_{3}$, the strangeness $(s)$, spin, and parity. In order to extract their masses in lattice QCD we evaluate two-point correlators. We use interpolating fields to create these states from the vacuum that have the correct quantum numbers and reduce to the quark model wave functions in the nonrelativistic limit. The

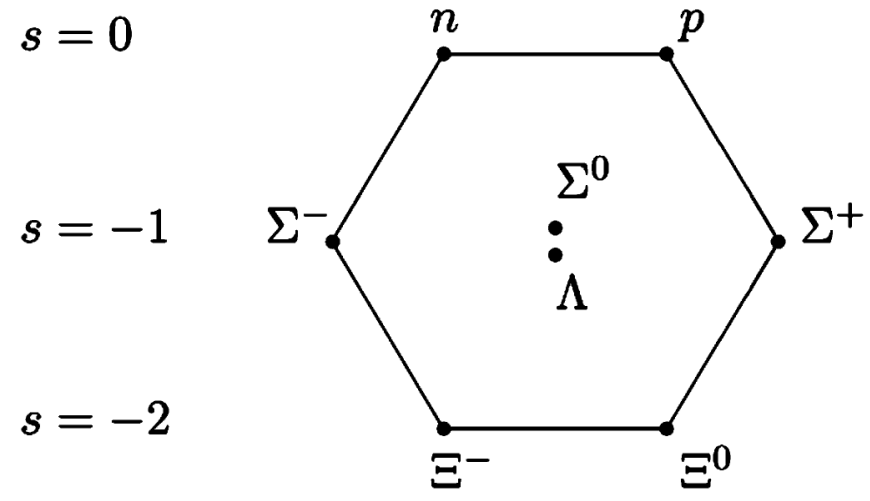

FIG. 1. The low-lying baryons belonging to the octet representation labeled by the value of $I_{3}$ and hypercharge.

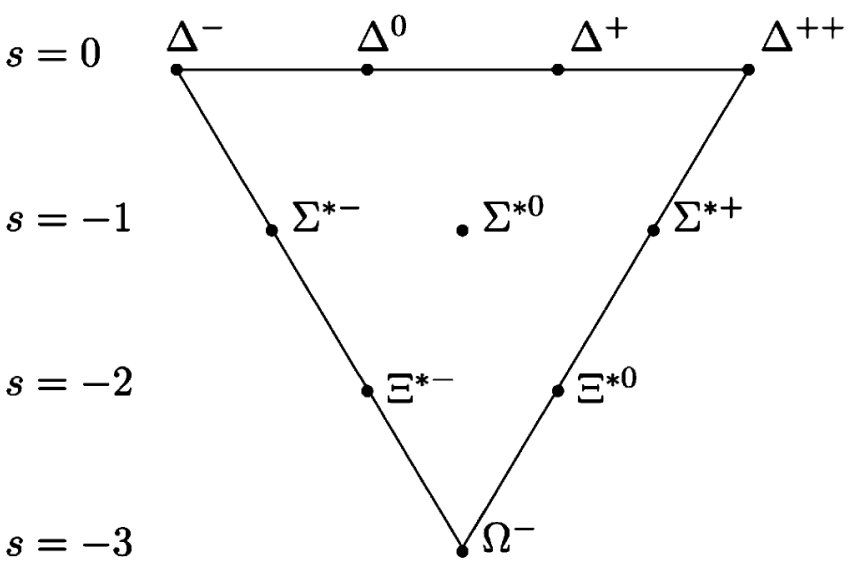

FIG. 2. The low-lying baryons belonging to the decuplet representation labeled by the value of $I_{3}$ and hypercharge.

interpolating fields used in this work are collected in Tables III [31,32] and IV [31,33] for the octet and decuplet, respectively.

Local interpolating fields are not optimal for suppressing excited state contributions. We instead apply Gaussian smearing to each quark field, $q(\mathbf{x}, t): q^{\text {smear }}(\mathbf{x}, t)=$ $\sum_{\mathbf{y}} F(\mathbf{x}, \mathbf{y} ; U(t)) q(\mathbf{y}, t)$ using the gauge invariant smearing function

$$
F(\mathbf{x}, \mathbf{y} ; U(t))=(1+\alpha H)^{n}(\mathbf{x}, \mathbf{y} ; U(t)),
$$

constructed from the hopping matrix,

$$
H(\mathbf{x}, \mathbf{y} ; U(t))=\sum_{i=1}^{3}\left(U_{i}(\mathbf{x}, t) \delta_{\mathbf{x}, \mathbf{y}-i}+U_{i}^{\dagger}(\mathbf{x}-i, t) \delta_{\mathbf{x}, \mathbf{y}+i}\right) .
$$

Furthermore we apply APE smearing [34] to the spatial links that enter the hopping matrix. The parameters of the Gaussian and APE smearing are the same as those used in our previous work devoted to the nucleon and $\Delta$ masses [4].

\section{E. Two-point correlators}

To extract masses in the rest frame we consider twopoint correlators defined by

$$
\begin{aligned}
C_{\bar{X}}^{ \pm}(t, \vec{p}=\overrightarrow{0})= & \frac{1}{2} \operatorname{Tr}\left(1 \pm \gamma_{4}\right) \\
& \times \sum_{\mathbf{x}_{\text {sink }}}\left\langle J_{X}\left(\mathbf{x}_{\text {sink }}, t_{\text {sink }}\right) \bar{J}_{X}\left(\mathbf{x}_{\text {source }}, t_{\text {source }}\right)\right\rangle, \\
t= & t_{\text {sink }}-t_{\text {source }} .
\end{aligned}
$$

Space-time reflection symmetries of the action and the antiperiodic boundary conditions in the temporal direction for the quark fields imply, for zero three-momentum correlators, that $C_{X}^{+}(t)=-C_{X}^{-}(T-t)$. So, in order to decrease errors we average correlators in the forward and 
TABLE III. Interpolating fields and quantum numbers for the baryons in the octet representation.

\begin{tabular}{|c|c|c|c|c|}
\hline Strangeness & Baryon & Interpolating field & $I$ & $I_{z}$ \\
\hline$s=0$ & $\begin{array}{l}p \\
n\end{array}$ & $\begin{array}{l}\chi^{p}=\epsilon_{a b c}\left(u_{a}^{T} C \gamma_{5} d_{b}\right) u_{c} \\
\chi^{n}=\epsilon_{a b c}\left(d_{a}^{T} C \gamma_{5} u_{b}\right) d_{c}\end{array}$ & $\begin{array}{l}1 / 2 \\
1 / 2\end{array}$ & $\begin{array}{l}+1 / 2 \\
-1 / 2\end{array}$ \\
\hline$s=1$ & $\begin{array}{l}\Lambda \\
\Sigma^{+} \\
\Sigma^{0} \\
\Sigma^{-}\end{array}$ & 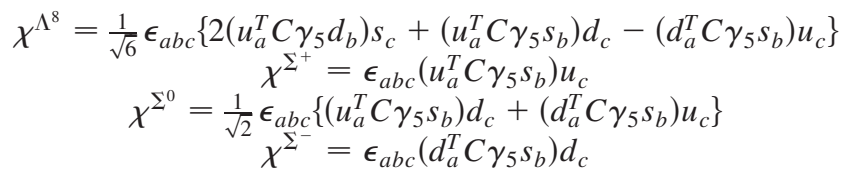 & $\begin{array}{l}0 \\
1 \\
1 \\
1\end{array}$ & $\begin{array}{l}0 \\
+1 \\
+0 \\
-1\end{array}$ \\
\hline$s=2$ & $\begin{array}{l}\Xi^{0} \\
\Xi^{-}\end{array}$ & $\begin{array}{l}\chi^{\Xi^{0}}=\epsilon_{a b c}\left(s_{a}^{T} C \gamma_{5} u_{b}\right) s_{c} \\
\chi^{\Xi^{-}}=\epsilon_{a b c}\left(s_{a}^{T} C \gamma_{5} d_{b}\right) s_{c}\end{array}$ & $\begin{array}{l}1 / 2 \\
1 / 2\end{array}$ & $\begin{array}{l}+1 / 2 \\
-1 / 2 \\
\end{array}$ \\
\hline
\end{tabular}

TABLE IV. Interpolating fields and quantum numbers for baryons in the decuplet representation.

\begin{tabular}{|c|c|c|c|c|}
\hline Strangeness & Baryon & Interpolating field & $I$ & $I_{z}$ \\
\hline \multirow{4}{*}{$s=0$} & $\Delta^{++}$ & $\chi_{\mu}^{\Delta^{++}}=\epsilon_{a b c}\left(u_{a}^{T} C \gamma_{\mu} u_{b}\right) u_{c}$ & $3 / 2$ & $+3 / 2$ \\
\hline & $\Delta^{+}$ & $\chi_{\mu}^{\Delta^{+}}=\frac{1}{\sqrt{3}} \epsilon_{a b c}\left\{2\left(u_{a}^{T} C \gamma_{\mu} d_{b}\right) u_{c}+\left(u_{a}^{T} C \gamma_{\mu} u_{b}\right) d_{c}\right\}$ & $3 / 2$ & $+1 / 2$ \\
\hline & $\Delta^{0}$ & $\chi_{\mu}^{\Delta^{0}}=\frac{1}{\sqrt{3}} \epsilon_{a b c}\left\{2\left(d_{a}^{T} C \gamma_{\mu} u_{b}\right) d_{c}+\left(d_{a}^{T} C \gamma_{\mu} d_{b}\right) u_{c}\right\}$ & $3 / 2$ & $-1 / 2$ \\
\hline & $\Delta^{-}$ & $\chi_{\mu}^{\Delta^{-}}=\epsilon_{a b c}\left(d_{a}^{T} C \gamma_{\mu} d_{b}\right) d_{c}$ & $3 / 2$ & $-3 / 2$ \\
\hline \multirow{3}{*}{$s=1$} & $\Sigma^{*+}$ & $\chi_{\mu}^{\Sigma_{\mu}^{*+}}=\frac{1}{\sqrt{3}} \epsilon_{a b c}\left\{\left(u_{a}^{T} C \gamma_{\mu} u_{b}\right) s_{c}+2\left(s_{a}^{T} C \gamma_{\mu} u_{b}\right) u_{c}\right\}$ & 1 & +1 \\
\hline & $\Sigma^{* 0}$ & $\chi_{\mu}^{\Sigma_{\mu}^{* 0}}=\sqrt{\frac{2}{3}} \epsilon_{a b c}\left\{\left(u_{a}^{T} C \gamma_{\mu} d_{b}\right) s_{c}+\left(d_{a}^{T} C \gamma_{\mu} s_{b}\right) u_{c}+\left(s_{a}^{T} C \gamma_{\mu} u_{b}\right) d_{c}\right\}$ & 1 & +0 \\
\hline & $\Sigma^{*-}$ & $\chi_{\mu}^{\Sigma^{*-}}=\frac{1}{\sqrt{3}} \epsilon_{a b c}\left\{\left(d_{a}^{T} C \gamma_{\mu} d_{b}\right) s_{c}+2\left(s_{a}^{T} C \gamma_{\mu} d_{b}\right) d_{c}\right\}$ & 1 & -1 \\
\hline \multirow{2}{*}{$s=2$} & $\Xi^{* 0}$ & $\chi \bar{\Xi}_{\mu}^{\Xi^{* 0}}=\epsilon_{a b c}\left(s_{a}^{T} C \gamma_{\mu} u_{b}\right) s_{c}$ & $1 / 2$ & $+1 / 2$ \\
\hline & $\Xi^{*-}$ & $\chi \ddot{\Xi}^{*-}=\epsilon_{a b c}\left(s_{a}^{T} C \gamma_{\mu} d_{b}\right) s_{c}$ & $1 / 2$ & $-1 / 2$ \\
\hline$s=3$ & $\Omega^{-}$ & $\chi_{\mu}^{\Omega^{-}}=\epsilon_{a b c}\left(s_{a}^{T} C \gamma_{\mu} s_{b}\right) s_{c}$ & 0 & +0 \\
\hline
\end{tabular}

backward directions and define

$$
C_{X}(t)=C_{X}^{+}(t)-C_{X}^{-}(T-t)
$$

In order to decrease correlation between measurements, we choose the source location randomly on the whole lattice for each configuration. Masses are extracted from the so-

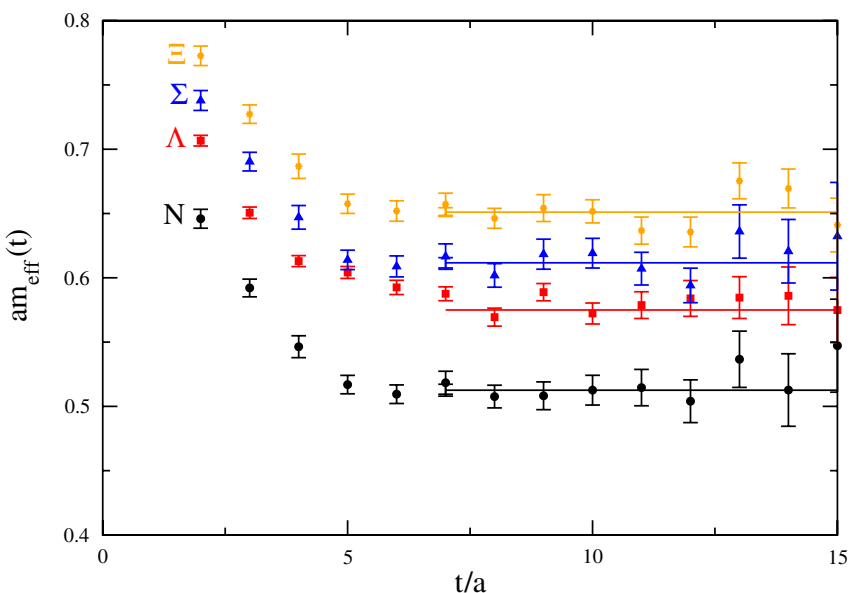

FIG. 3 (color online). Effective masses of the octet states for $\beta=3.9, a \mu=0.004$ on a $32^{3} \times 64$ lattice using 232 configurations. called effective mass which is defined by

$$
\begin{aligned}
a m_{\mathrm{eff}}^{X}(t) & =-\log \left(C_{X}(t) / C_{X}(t-1)\right) \\
& =a m_{X}+\log \left(\frac{1+\sum_{i=1}^{\infty} c_{i} e^{\Delta_{i} t}}{1+\sum_{i=1}^{\infty} c_{i} e^{\Delta_{i}(t-1)}}\right) \underset{t \rightarrow \infty}{\rightarrow} a m_{X},
\end{aligned}
$$

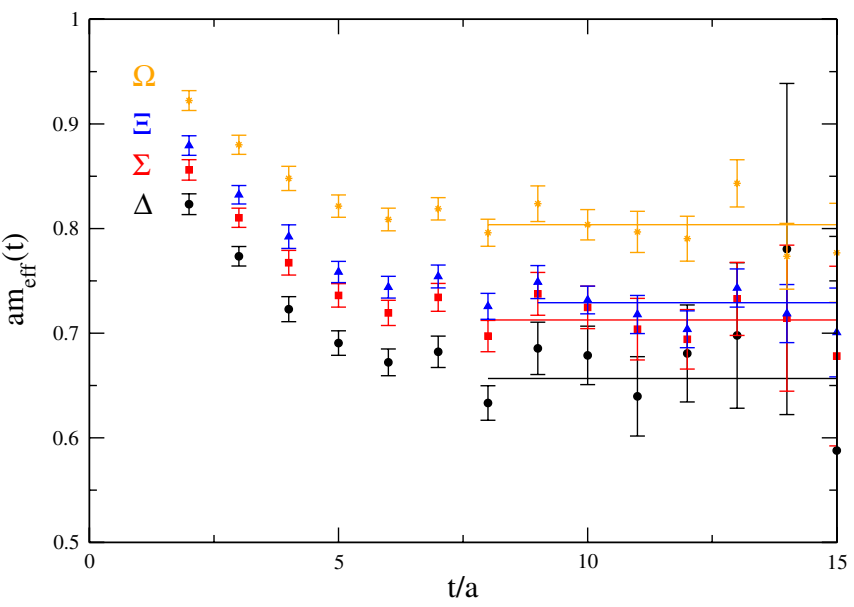

FIG. 4 (color online). Effective masses of the decuplet states for $\beta=3.9, a \mu=0.004$ on a $32^{3} \times 64$ lattice using 232 configurations. 
TABLE V. Baryon masses in the octet representation at $\beta=3.9$ in lattice units.

\begin{tabular}{ccccccccccc}
\hline \hline$a \mu$ & stat. & $a m_{N}$ & $a m_{\Lambda}$ & $a m_{\Sigma^{\mathrm{Av}}}$ & $a m_{\Sigma^{+}}$ & $a m_{\Sigma^{0}}$ & $a m_{\Sigma^{-}}$ & $a m_{\Xi^{\mathrm{Av}}}$ & $a m_{\Xi^{0}}$ & $a m_{\Xi^{-}}$ \\
\hline 0.0040 & 782 & $0.5111(58)$ & $0.5787(42)$ & $0.6075(46)$ & $0.6175(66)$ & $0.6118(48)$ & $0.5959(52)$ & $0.6497(31)$ & $0.6695(42)$ & $0.6372(31)$ \\
0.0064 & 545 & $0.5514(49)$ & $0.6017(42)$ & $0.6265(48)$ & $0.6487(72)$ & $0.6278(52)$ & $0.6131(52)$ & $0.6636(36)$ & $0.6876(50)$ & $0.6500(36)$ \\
0.0085 & 348 & $0.5786(67)$ & $0.6198(51)$ & $0.6491(55)$ & $0.6679(62)$ & $0.6529(49)$ & $0.6358(46)$ & $0.6728(43)$ & $0.6956(58)$ & $0.6593(43)$ \\
0.0100 & 477 & $0.5973(43)$ & $0.6326(36)$ & $0.6522(41)$ & $0.6662(56)$ & $0.6539(43)$ & $0.6429(44)$ & $0.6793(36)$ & $0.6959(49)$ & $0.6683(32)$ \\
0.0030 & 652 & $0.4958(43)$ & $0.5613(33)$ & $0.5891(42)$ & $0.6069(68)$ & $0.5932(50)$ & $0.5775(39)$ & $0.6382(30)$ & $0.6572(44)$ & $0.6275(26)$ \\
0.0040 & 232 & $0.5126(46)$ & $0.5750(35)$ & $0.6117(40)$ & $0.6281(73)$ & $0.6158(40)$ & $0.5960(47)$ & $0.6511(34)$ & $0.6748(46)$ & $0.6358(32)$ \\
\hline \hline
\end{tabular}

TABLE VI. Baryon masses in the decuplet representation at $\beta=3.9$ in lattice units.

\begin{tabular}{|c|c|c|c|c|c|c|c|c|c|c|c|}
\hline$a \mu$ & stat. & $a m_{\Delta^{++,-}}$ & $a m_{\Delta^{+, 0}}$ & $a m_{\sum^{* A v}}$ & $a m_{\sum^{*+}}$ & $a m_{\Sigma^{* 0}}$ & $a m_{\Sigma^{*-}}$ & $a m_{\Xi^{* \mathrm{Av}}}$ & $a m_{\Xi^{* 0}}$ & $a m_{\Xi^{*-}}$ & $a m_{\Omega}$ \\
\hline & & & & & & $24^{3} \times 48$ & & & & & \\
\hline 0.0040 & 782 & $0.660(14)$ & $0.670(13)$ & $0.7166(82)$ & $0.709(11)$ & $0.7226(81)$ & $0.7222(95)$ & $0.7311(51)$ & $0.7381(59)$ & $0.7200(66)$ & $0.8079(52)$ \\
\hline 0.0064 & 545 & $0.709(11)$ & $0.711(12)$ & $0.7461(84)$ & $0.740(10)$ & $0.7480(93)$ & $0.7489(93)$ & $0.7412(78)$ & $0.7552(76)$ & $0.7344(84)$ & $0.8156(63)$ \\
\hline 0.0085 & 348 & $0.714(12)$ & $0.733(13)$ & $0.7517(88)$ & $0.739(11)$ & $0.760(11)$ & $0.7645(98)$ & $0.7415(85)$ & $0.7529(81)$ & $0.7367(84)$ & $0.8133(66)$ \\
\hline 0.0100 & 477 & $0.7531(67)$ & $0.7559(75)$ & $0.7794(66)$ & $0.7808(62)$ & $\begin{array}{l}0.7809(64) \\
32^{3} \times 64\end{array}$ & $0.7798(69)$ & $0.7618(73)$ & $0.7741(64)$ & $0.7484(74)$ & $0.8284(51)$ \\
\hline 0.003 & 652 & $0.6234(139)$ & $0.6497(133)$ & $0.6859(96)$ & $0.6838(93)$ & $0.6859(106)$ & $0.7027(101)$ & & & $0.7032(53)$ & $0.7926(49)$ \\
\hline 0.004 & 232 & $0.651(16)$ & $0.659(15)$ & $0.713(10)$ & $0.705(12)$ & $0.716(12)$ & $0.7173(99)$ & $0.7291(74)$ & $0.7366(79)$ & $0.7192(72)$ & $0.8037(69)$ \\
\hline
\end{tabular}

where $\Delta_{i}=m_{i}-m_{X}$ is the mass difference of the excited state $i$ with respect to the ground state mass $m_{X}$.

In Figs. 3 and 4 we show the effective masses of the baryons in the octet and decuplet representation, respectively. As can be seen a plateau region can be identified for all baryons. What is shown in these figures are effective masses extracted from correlators where smearing is applied both at the sink and at the source. Although local correlators are expected to have the same value in the large time limit, smearing suppresses excited state contributions yielding a plateau at earlier time separations and a better accuracy in the mass extraction. Our fitting procedure to extract $m_{X}$ is as follows: The mass is obtained from the leading term in Eq. (15), i.e. from a constant fit to $m_{X}$. A second fit, including the first excited state, allows us to estimate the systematic error of the previously determined $m_{X}$ due to the first excited state for a given plateau range. The plateau range is then chosen such that the systematic error on $m_{X}$ drops below $50 \%$ of its statistical error This criterion is in most of the cases in agreement with a $\chi^{2} /$ d.o.f. $<1$. In the cases in which this criterion is not satisfied a careful examination of the effective mass is made to ensure that the fit range is in the plateau region. The results for the masses of the octet and decuplet at

TABLE VII. Baryon masses in the octet representation at $\beta=4.05$ in lattice units.

\begin{tabular}{ccccccccccc}
\hline \hline$a \mu$ & stat. & $a m_{N}$ & $a m_{\Lambda}$ & $a m_{\Sigma^{a v}}$ & $a m_{\Sigma^{+}}$ & $a m_{\Sigma^{0}}$ & $a m_{\Sigma^{-}}$ & $a m_{\Xi^{a v}}$ & $a m_{\Xi^{0}}$ & $a m_{\Xi^{-}}$ \\
\hline 0.0030 & 269 & $0.4091(60)$ & $0.4540(38)$ & $0.4761(44)$ & $0.4885(62)$ & $0.4774(47)$ & $0.4651(53)$ & $0.5082(31)$ & $0.5177(39)$ & $0.5007(29)$ \\
0.0060 & 253 & $0.4444(47)$ & $0.4792(47)$ & $0.4944(44)$ & $0.5022(66)$ & $0.4960(45)$ & $0.4834(45)$ & $0.5192(42)$ & $0.5277(50)$ & $0.5112(37)$ \\
0.0080 & 409 & $0.4714(31)$ & $0.4957(30)$ & $0.5089(31)$ & $0.5179(41)$ & $0.5095(32)$ & $0.5019(31)$ & $0.5262(28)$ & $0.5350(34)$ & $0.5199(25)$ \\
\hline \hline
\end{tabular}

TABLE VIII. Baryon masses in the decuplet representation at $\beta=4.05$ in lattice units.

\begin{tabular}{|c|c|c|c|c|c|c|c|c|c|c|c|}
\hline$a \mu$ & stat. & $a m_{\Delta^{++,-}}$ & $a m_{\Delta^{+, 0}}$ & $a m_{\Sigma^{* a v}}$ & $a m_{\Sigma^{*+}}$ & $a m_{\sum^{* 0}}$ & $a m_{\sum^{*-}}$ & $a m_{\Xi^{* a v}}$ & $a m_{\Xi^{* 0}}$ & $a m_{\Xi^{*-}}$ & $a m_{\Omega}$ \\
\hline & & & & & & $32^{3} \times 64$ & & & & & \\
\hline 0.0030 & 269 & $0.5381(93)$ & $0.5441(93)$ & $0.5728(79)$ & $0.5673(94)$ & 0.575 & $0.5734(80)$ & $0.5772(56)$ & $0.5796(54)$ & $0.5750(56)$ & 0.636 \\
\hline 0.0060 & 253 & $0.5505(77)$ & $0.5581(90)$ & $0.5805(66)$ & $0.5754(7$ & 0.581( & & 0.5816 & 0.5834 & 0.5802( & 0.6286 \\
\hline 0.0080 & 409 & $0.5918(60)$ & $0.5906(63)$ & $0.6078(59)$ & $0.6044(68)$ & $0.5850(74)$ & $0.6099(57)$ & $0.5940(43)$ & $0.6021(50)$ & $0.5873(43)$ & $0.6461(49)$ \\
\hline
\end{tabular}




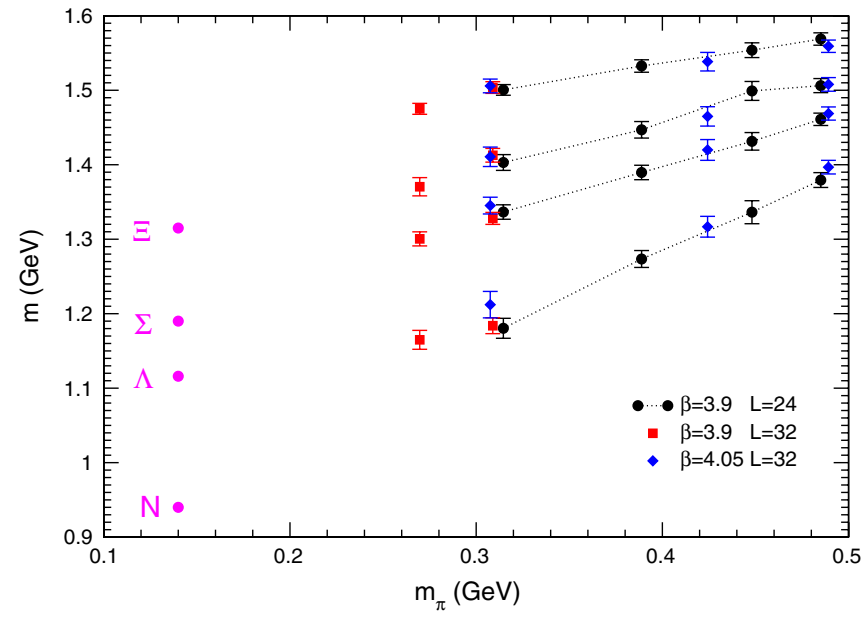

FIG. 5 (color online). Octet states measured in our different gauge ensembles. Physical points are indicated by their name symbols (filled circles). The data at $\beta=3.9, L / a=24$ are connected by dotted lines to guide the eye.

$\beta=3.9$ are collected in Tables V and VI, respectively. The corresponding results for the masses at $\beta=4.05$ are given in Tables VII and VIII. The errors are evaluated using both jackknife and the $\Gamma$ method [35] to check consistency.

\section{RESULTS}

The bulk of the numerical results is presented in this section. Baryon masses are given in lattice units. Our procedure to convert the results to physical units will be discussed in the next section.

\section{A. Baryon masses}

In Tables V, VI, VII, and VIII we present the masses of the octet and decuplet states with the lattice input parameters given in Table I. For the isospin multiplets we have computed separately the masses corresponding to each isospin component as well as their averaged value. These results (averaged values in case of isospin multiplets) are displayed in Figs. 5 and 6. The $\beta=3.9, L / a=24$ data are

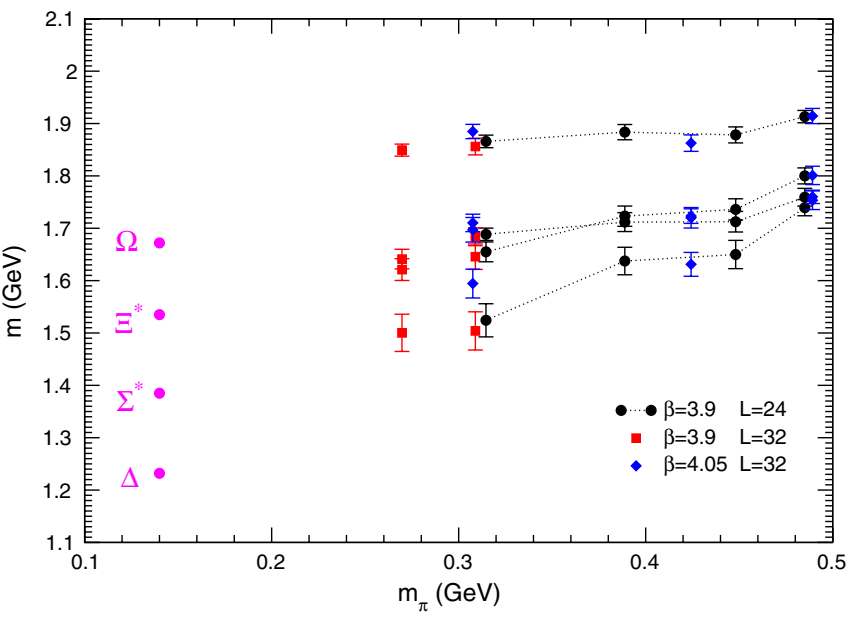

FIG. 6 (color online). The same as Fig. 5 but for the decuplet states.

linked by dotted lines to guide the eye. An inspection of the plots indicates that the lattice artifacts, studied in detail in the next section, are small. Notice that the natural order of the $\Sigma^{*}$ and $\Xi^{*}$ states comes out to be correct for $m_{\pi} \leq$ $300 \mathrm{MeV}$, while for larger masses this order is inverted.

\section{B. Strange quark mass dependence}

The dependence of the masses of baryons with strangeness on the bare strange quark mass has been investigated at $\beta=3.9$ for $a \mu=0.004$. The results are given in Tables IX and X and displayed in Figs. 7 and 8. The vertical dotted lines indicate the value of the tuned bare strange quark mass as given in Table II. The SU(3) symmetric point $\mu_{s}=\mu$ is given by the nucleon and $\Delta$ mass for the octet and decuplet, respectively. As can be seen in the SU(3) limit all the octet and decuplet masses converge to a single point. Possible cutoff effects and the fact that we only have $N_{f}=2$ simulations can account for the small deviations. For clarity we only show in Fig. 7 the mass of $\Lambda, \Sigma^{\mathrm{Av}}$, and $\Xi^{\mathrm{Av}}$. They should be degenerate with the nucleon in the limit of $\mu_{s}=\mu$. Indeed, if one computes the nucleon mass with the same statistics with that used for

TABLE IX. Octet masses for $\beta=3.9, a \mu=0.004$ on a $24^{3} \times 48$ lattice as a function of $a \mu_{s}$.

\begin{tabular}{|c|c|c|c|c|c|c|c|c|c|}
\hline$a \mu_{s}$ & stat. & $a m_{\Lambda}$ & $a m_{\sum^{A v}}$ & $a m_{\Sigma^{+}}$ & $a m_{\Sigma^{0}}$ & $a m_{\Sigma^{-}}$ & $a m_{\Xi^{\mathrm{Av}}}$ & $a m_{\Xi^{0}}$ & $a m_{\Xi^{-}}$ \\
\hline \multicolumn{10}{|c|}{$24^{3} \times 48$} \\
\hline 0.0064 & 597 & $0.533(8)$ & $0.545(7)$ & $0.549(12)$ & $0.563(5)$ & $0.537(6)$ & $0.545(7)$ & $0.560(11)$ & $0.530(6)$ \\
\hline 0.0085 & 316 & $0.537(10)$ & $0.557(11)$ & $0.559(19)$ & $0.557(12)$ & $0.554(8)$ & $0.563(9)$ & $0.585(14)$ & $0.549(8)$ \\
\hline 0.0100 & 316 & $0.542(9)$ & $0.564(10)$ & $0.567(18)$ & $0.564(11)$ & $0.561(7)$ & $0.574(8)$ & $0.597(13)$ & $0.560(7)$ \\
\hline 0.0175 & 315 & $0.563(8)$ & $0.596(9)$ & $0.600(14)$ & $0.593(9)$ & $0.593(7)$ & $0.626(6)$ & $0.644(8)$ & $0.610(6)$ \\
\hline 0.0200 & 308 & $0.568(8)$ & $0.606(8)$ & $0.609(13)$ & $0.602(9)$ & $0.603(6)$ & $0.641(6)$ & $0.660(8)$ & $0.625(5)$ \\
\hline 0.0250 & 311 & $0.584(7)$ & $0.626(6)$ & $0.627(12)$ & $0.619(8)$ & $0.620(6)$ & $0.671(5)$ & $0.688(7)$ & $0.656(5)$ \\
\hline 0.0400 & 316 & $0.624(7)$ & $0.674(6)$ & $0.676(10)$ & $0.667(7)$ & $0.672(6)$ & $0.751(4)$ & $0.764(5)$ & $0.738(4)$ \\
\hline 0.0800 & 314 & $0.718(7)$ & $0.780(5)$ & $0.787(7)$ & $0.772(7)$ & $0.776(7)$ & $0.935(3)$ & $0.945(4)$ & $0.926(3)$ \\
\hline
\end{tabular}


TABLE X. Decuplet masses for $\beta=3.9, a \mu=0.004$ on a $24^{3} \times 48$ lattice as a function of $a \mu_{s}$.

\begin{tabular}{|c|c|c|c|c|c|c|c|c|c|}
\hline$a \mu_{s}$ & stat. & $a m_{\sum^{* A v}}$ & $a m_{\Sigma^{*+}}$ & $a m_{\sum^{* 0}}$ & $a m_{\Sigma^{*-}}$ & $a m_{\Xi^{* \mathrm{Av}}}$ & $a m_{\Xi^{* 0}}$ & $a m_{\Xi^{*-}}$ & $a m_{\Omega}$ \\
\hline \multicolumn{10}{|c|}{$24^{3} \times 48$} \\
\hline 0.0064 & 597 & $0.665(12)$ & $0.658(18)$ & $0.669(14)$ & $0.669(14)$ & $0.636(9)$ & $0.645(12)$ & $0.628(9)$ & $0.678(14)$ \\
\hline 0.0085 & 316 & $0.695(19)$ & $0.719(13)$ & $0.713(16)$ & $0.733(9)$ & $0.648(11)$ & $0.670(15)$ & $0.624(10)$ & $0.734(11)$ \\
\hline 0.0100 & 316 & $0.700(18)$ & $0.722(12)$ & $0.715(15)$ & $0.697(22)$ & $0.658(9)$ & $0.680(13)$ & $0.636(9)$ & $0.744(10)$ \\
\hline 0.0175 & 315 & $0.721(14)$ & $0.729(14)$ & $0.736(12)$ & $0.718(19)$ & $0.705(7)$ & $0.722(8)$ & $0.690(7)$ & $0.796(6)$ \\
\hline 0.0200 & 308 & $0.725(14)$ & $0.734(13)$ & $0.741(11)$ & $0.718(17)$ & $0.720(6)$ & $0.734(7)$ & $0.704(7)$ & $0.807(7)$ \\
\hline 0.0250 & 311 & $0.740(13)$ & $0.735(16)$ & $0.753(11)$ & $0.740(17)$ & $0.747(6)$ & $0.759(6)$ & $0.733(6)$ & $0.838(6)$ \\
\hline 0.0400 & 316 & $0.778(11)$ & $0.770(13)$ & $0.788(9)$ & $0.778(15)$ & $0.821(5)$ & $0.831(5)$ & $0.811(5)$ & $0.934(4)$ \\
\hline 0.0800 & 314 & $0.864(7)$ & $0.854(11)$ & $0.870(8)$ & $0.865(9)$ & $0.993(4)$ & $0.996(5)$ & $0.987(4)$ & $1.169(3)$ \\
\hline
\end{tabular}

$\Sigma^{\mathrm{Av}}$ and $\Xi^{\mathrm{Av}}$, one finds them to be degenerate within the errors as can be seen in Fig. 7.

The corresponding results for the decuplet baryons are displayed in Fig. 8. As can be seen, also in the case of the decuplet masses there is convergence to the $\Delta$ mass as predicted in the exact $\mathrm{SU}(3)$ limit $\mu_{s}=\mu$.

The $\mu_{s}$ dependence of the strange baryon masses provides an estimate of systematic errors due to the uncertainty in the tuning of the strange quark mass. As already explained, the kaon mass at the physical point is used to fix $\mu_{s}$. This gives $a \mu_{s}=0.0217(22)$. The $\sim 10 \%$ uncertainty leads to a corresponding error in the strange baryon masses that can be estimated by the variation of their masses in the vicinity of $\mu_{s}$. At $\mu=0.004$ we estimate an error that is comparable to the statistical error. In what follows we will analyze our results taking into account only statistical errors. This analysis shows that when the statistical error is given on the final results of strangeness nonzero baryon masses one must bear in mind that there is a systematic error of about the same magnitude due to the strange quark mass determination.

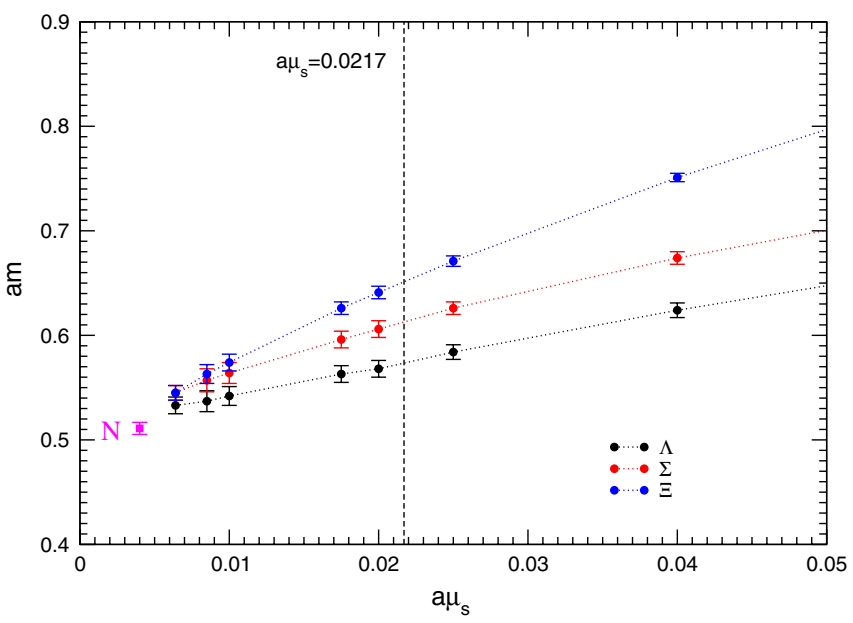

FIG. 7 (color online). Masses for octet baryons at $\beta=3.9$ and $a \mu=0.004$ on a lattice of size $24^{3} \times 48$ as a function of $a \mu_{s}$. The vertical dashed line indicates the value of the tuned bare strange quark mass. The dotted lines are to guide the eye.

\section{Gell-Mann-Okubo relation}

Assuming a small SU(3) breaking, Okubo derived interesting relations among baryon masses. We examine in this section how well the GMO relations [36] are fulfilled for the baryon masses obtained on our lattices at different pion mass values. As we will discuss in detail in the next section, volume and discretization effects are small, and therefore it suffices to analyze the $\beta=3.9$ and $L=24 \times$ 48 results. For this study we use the lattice spacing determined from $f_{\pi}$ to convert to physical units.

For the $J^{P}=1 / 2^{+}$octet the GMO relation can be written in the form:

$$
\frac{M_{\Xi}+M_{N}}{2}=\frac{3 M_{\Lambda}+M_{\Sigma}}{4} .
$$

The results are displayed in Fig. 9 where the left- and righthand side terms of Eq. (16) are separately plotted as a function of $m_{\pi}^{2}$. The difference between the two terms is compatible with zero at any pion mass. The experimental values, shown by the squares, are, respectively, 254 and $248 \mathrm{MeV}$. These results are similar to those presented in Ref. [37] using a mixed action setup with valence domain wall fermions on rooted staggered sea fermions.

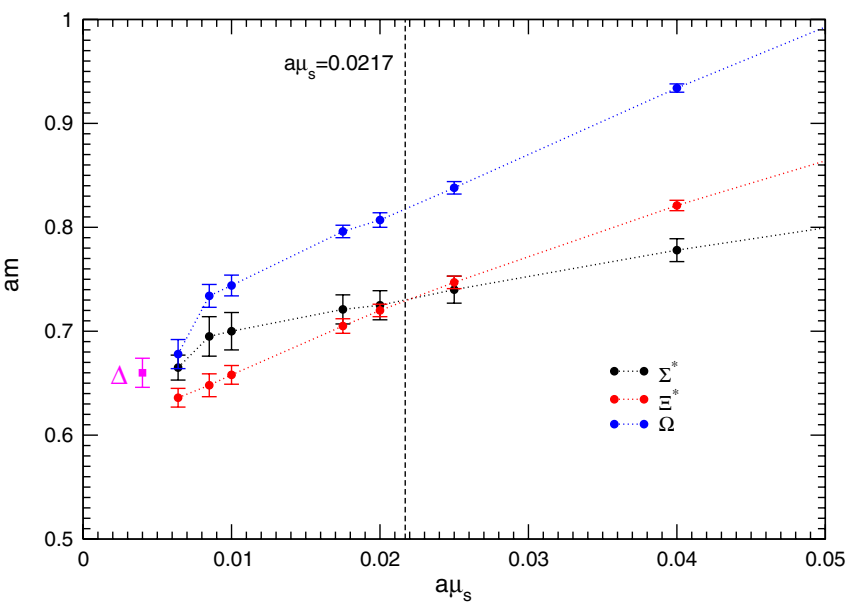

FIG. 8 (color online). The same as for Fig. 7 but for the decuplet baryons. 

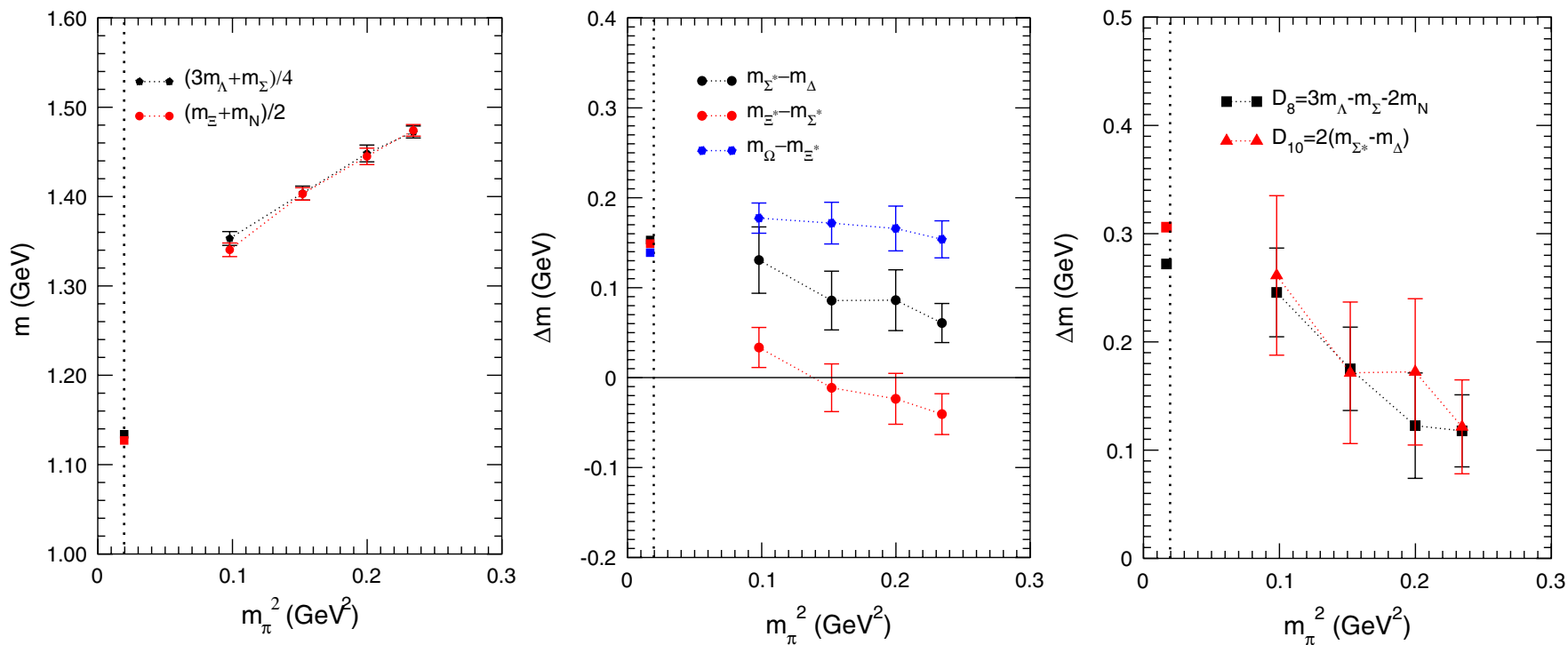

FIG. 9 (color online). Gell-Mann-Okubo relations for the baryon octet (left panel), decuplet (middle panel), and mixed octet decuplet (right panel) as a function of $m_{\pi}^{2}$. Vertical lines correspond to the physical results. Data were obtained from simulations at $\beta=3.9$ and $L / a=24$. The lattice spacing determined from $f_{\pi}$ is used to convert to physical units.

For the $J^{P}=3 / 2^{+}$decuplet, the GMO relations predict equal mass difference among two consecutive $(\Delta S=1)$ isospin multiplets:

$$
M_{\Sigma^{*}}-M_{\Delta}=M_{\Xi^{*}}-M_{\Sigma^{*}}=M_{\Omega}-M_{\Xi^{*}} .
$$

The results for the decuplet baryons are displayed in Fig. 9. As can be seen, the equalities of Eq. (17) are strongly violated; the three mass differences of Eq. (17) are spread over about $200 \mathrm{MeV}$ for the range of pion masses that have been computed. The experimental values for these mass differences are 153, 149, and $139 \mathrm{MeV}$, shown in the plot by the squares. In the lattice results the larger deviation comes from $M_{\Xi^{*}}-M_{\Sigma^{*}}$, while for $M_{\Sigma^{*}}-M_{\Delta}$ and $M_{\Omega}-$ $M_{\Xi^{*}}$ the mass differences are smaller. The mass difference $M_{\Xi^{*}}-M_{\Sigma^{*}}$ is increasing as the pion mass decreases. Unfortunately, with our present statistics it is unclear whether this increase is sufficient to bring this mass difference in agreement with experiment but the trend is definitely in the right direction.

A third relation exists that connects the $J^{P}=1 / 2^{+}$octet masses with the $J^{P}=3 / 2^{+}$decuplet masses, which reads as

$$
3 M_{\Lambda}-M_{\Sigma}-2 M_{N}=2\left(M_{\Sigma^{*}}-M_{\Delta}\right) .
$$

Experimentally, this relation is fulfilled at the $10 \%$ level yielding $276 \mathrm{MeV}$ for the left-hand side and $305 \mathrm{MeV}$ for the right-hand side of Eq. (18). These values are shown by the filled squares in Fig. 9. The corresponding lattice results are shown in the same figure. One can see that, as in the octet case, the relation of Eq. (18) is satisfied within our statistical uncertainties at each pion mass. It also approaches the experimental results with decreasing pion mass.

Fulfillment of the GMO relations is considered a success of SU(3) symmetry. Therefore one would expect that these relations are better satisfied as we approach the SU(3) limit $a \mu=a \mu_{s}=0.0217$, up to discretization effects. This corresponds to about $m_{\pi}^{2} \sim 0.50 \mathrm{GeV}^{2}$. For the decuplet mass relation given in Eq. (17) it is unclear if this would be indeed satisfied by the lattice data, whereas the other two relations are fulfilled at all masses.

\section{SYSTEMATICS}

In order to compare our lattice results collected in Tables V, VI, VII, and VIII, to the physical masses we need to check for finite volume and cutoff effects, and carry out the extrapolation to the physical light quark mass. The strange quark was fixed to the physical value using the kaon mass with the light quarks extrapolated to the physical point as explained in Sec. II B. A check of the effect of this tuning on baryon masses has been discussed in Sec. III B. In this section we discuss finite volume and cutoff effects, in particular, the isospin breaking.

\section{A. Finite volume effects}

Finite volume corrections to the nucleon mass in $N_{f}=2$ lattice QCD have been studied in Ref. [5] within the $p$ expansion which assumes that finite size effects originate from pions that propagate around the spatial box. Using relativistic $\mathrm{SU}(2)$ baryon chiral perturbation theory $(\chi \mathrm{PT})$ [38], the finite volume corrections to the nucleon mass to $\mathcal{O}\left(p^{4}\right)$ are 


$$
m_{N}(\infty)=m_{N}(L)-\delta m_{a}(L)-\delta m_{b}(L),
$$

where

$$
\begin{aligned}
\delta m_{a}(L)= & \frac{3 g_{A}^{2} m_{N}^{0} m_{\pi}^{2}}{8 \pi^{2} f_{\pi}^{2}} \int_{0}^{\infty} d x \\
& \times \sum_{\mathbf{n}}^{\prime} K_{0}\left(L|\mathbf{n}| \sqrt{\left(m_{N}^{0}\right)^{2} x^{2}+m_{\pi}^{2}(1-x)}\right), \\
\delta m_{b}(L)= & \frac{3 m_{\pi}^{4}}{2 \pi^{2} f_{\pi}^{2}} \sum_{\mathbf{n}}^{\prime}\left[\left(2 c_{1}-c_{3}\right) \frac{K_{1}\left(L|\mathbf{n}| m_{\pi}\right)}{L|\mathbf{n}| m_{\pi}}\right. \\
& \left.+c_{2} \frac{K_{2}\left(L|\mathbf{n}| m_{\pi}\right)}{\left(L|\mathbf{n}| m_{\pi}\right)^{2}}\right] .
\end{aligned}
$$

$K_{\nu}(x)$ is the modified Bessel function and the sum is over all integer vectors $\mathbf{n}$ excluding $\mathbf{n}=\mathbf{0}$. The parameters $m_{N}^{0}$ and $c_{1}$ are determined by fitting first the nucleon mass to the same order [39-41] given by

$$
\begin{aligned}
m_{N}= & m_{N}^{0}-4 c_{1} m_{\pi}^{2}-\frac{3 g_{A}^{2}}{16 \pi f_{\pi}^{2}} m_{\pi}^{3}-4 E_{1}(\lambda) m_{\pi}^{4} \\
& +\frac{3 m_{\pi}^{4}}{16 \pi^{2} f_{\pi}^{2}}\left[\frac{1}{4}\left(c_{2}-\frac{2 g_{A}^{2}}{m_{N}^{0}}\right)\right. \\
& \left.-\left(c_{2}-8 c_{1}+4 c_{3}+\frac{g_{A}^{2}}{m_{N}^{0}}\right) \log \left(\frac{m_{\pi}}{\lambda}\right)\right] .
\end{aligned}
$$

We take the cutoff scale $\lambda=1 \mathrm{GeV}, f_{\pi}=130.70 \mathrm{MeV}$ and fix the dimension two low energy constants $c_{2}=$ $3.2 \mathrm{GeV}^{-1}$ [42] and $c_{3}=-3.45 \mathrm{GeV}^{-1}$ [40,43]. These values are consistent with empirical nucleon-nucleon phase shifts $[44,45]$. The counterterm $E_{1}$ is taken as an additional fit parameter. Using the values for $c_{1}$ and $m_{N}^{0}$ determined from the fit to the nucleon mass we estimate the volume corrections given in Table XI. As can be seen the corrections for our lattices are, in all cases except one, smaller than the statistical errors. In the analysis that follows we will use the volume corrected nucleon mass.

Concerning the other baryons a recent analysis using SU(3) heavy baryon chiral perturbation theory has shown

TABLE XI. Volume correction to the nucleon mass.

\begin{tabular}{llc}
\hline \hline$a m_{\pi}$ & $a m_{N}(L)$ & $a \delta_{a}(L)+a \delta_{b}(L)$ \\
\hline \multicolumn{3}{c}{$\beta=3.9,24^{3} \times 48$} \\
0.1362 & $0.5111(58)$ & 0.0068 \\
0.1684 & $0.5514(49)$ & 0.0046 \\
0.1940 & $0.5786(67)$ & 0.0026 \\
0.2100 & $0.5973(43)$ & 0.0021 \\
0.1168 & $\beta=3.9,32^{3} \times 64$ & \\
0.1338 & $0.4958(34)$ & 0.0014 \\
& $0.5126(46)$ & 0.0011 \\
0.1038 & $\beta=4.05,32^{3} \times 64$ & \\
0.1432 & $0.4091(60)$ & 0.0035 \\
0.1651 & $0.4444(47)$ & 0.0018 \\
\hline \hline
\end{tabular}

that the volume corrections are smaller than for the nucleon [46]. Given that the volume corrections found for the nucleon are smaller than the statistical errors we can safely neglect any volume corrections for the other baryons computed in this work. This is also corroborated by our lattice results at $a \mu=0.004$ where simulations at two volumes are used.

\section{B. Isospin breaking}

The twisted mass action breaks isospin explicitly to $\mathcal{O}\left(a^{2}\right)$. How large this breaking is depends on the size of the $\mathcal{O}\left(a^{2}\right)$ terms. It was shown that this cutoff effect is particularly large for the neutral pion [15] but small for other quantities. Indeed we verified that isospin breaking between the $\Delta^{++, 0}$ and $\Delta^{+,-}$is consistent with zero for lattice spacings below about $0.1 \mathrm{fm}$ [4]. We here address this issue for the octet and decuplet baryons. We show in Fig. 10 the mass differences for the $\Sigma, \Xi, \Delta, \Sigma^{*}$, and $\Xi^{*}$ charge multiplets as a function of the pion mass at two values of $\beta$.

As can be seen, we confirm that for the $\Delta$ system isospin breaking is consistent with zero. This is also true for the $\Sigma^{*}$

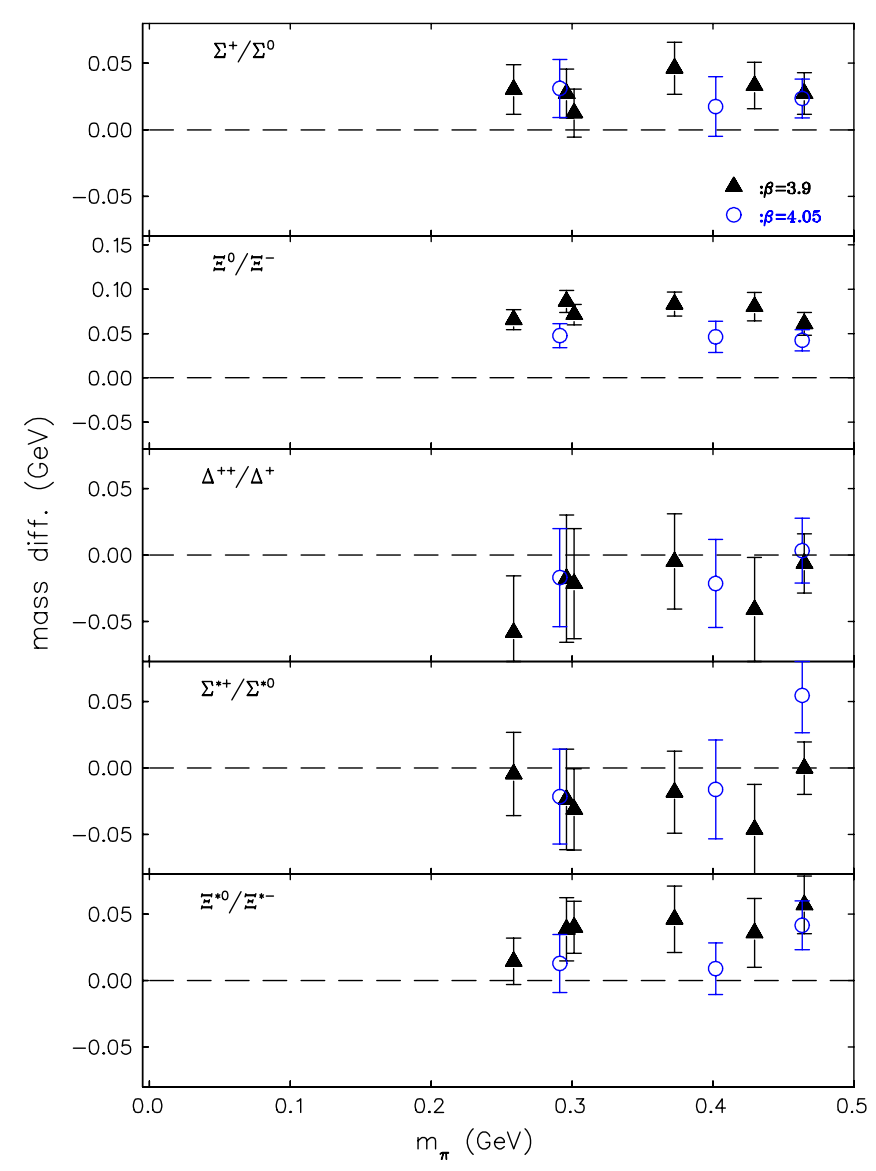

FIG. 10 (color online). Mass splitting versus the pion mass at $\beta=3.9$ (filled triangles) and $\beta=4.05$ (open circles) from top to bottom: $\Sigma^{+}$and $\Sigma^{0}, \Xi^{0}$ and $\Xi^{-}, \Delta^{++}$and $\Delta^{+}, \Sigma^{*+}$ and $\Sigma^{* 0}$, and $\Xi^{* 0}$ and $\Xi^{*-}$. 
TABLE XII. Octet masses computed at reference pion masses in units of $r_{0}$ at $\beta=3.9$ and $\beta=4.05$ and at the continuum limit.

\begin{tabular}{|c|c|c|c|c|c|c|c|c|c|}
\hline$r_{0} m_{\pi}$ & $r_{0} m_{N}$ & $r_{0} m_{\Lambda}$ & $r_{0} m_{\sum^{\mathrm{Av}}}$ & $r_{0} m_{\Sigma^{+}}$ & $r_{0} m_{\Sigma^{0}}$ & $r_{0} m_{\Sigma^{-}}$ & $r_{0} m_{\Xi^{\mathrm{Av}}}$ & $r_{0} m_{\Xi^{0}}$ & $r_{0} m_{\Xi^{-}}$ \\
\hline \multicolumn{10}{|c|}{$\beta=3.9$} \\
\hline 0.60 & $2.571(23)$ & $2.922(18)$ & $3.062(22)$ & $3.156(36)$ & $3.084(26)$ & $3.004(21)$ & $3.324(16)$ & $3.421(23)$ & $3.271(14)$ \\
\hline 0.70 & $2.671(24)$ & $3.001(18)$ & $3.193(21)$ & $3.279(38)$ & $3.215(21)$ & $3.111(25)$ & $3.399(18)$ & $3.523(24)$ & $3.319(17)$ \\
\hline 0.80 & $2.757(30)$ & $3.085(25)$ & $3.212(29)$ & $3.318(42)$ & $3.219(31)$ & $3.144(31)$ & $3.433(21)$ & $3.555(30)$ & $3.363(21)$ \\
\hline 0.90 & $2.880(26)$ & $3.156(22)$ & $3.286(25)$ & $3.404(38)$ & $3.293(27)$ & $3.215(27)$ & $3.472(19)$ & $3.598(26)$ & $3.401(19)$ \\
\hline 1.00 & $2.992(35)$ & $3.226(27)$ & $3.381(29)$ & $3.482(33)$ & $3.401(26)$ & $3.310(24)$ & $3.507(23)$ & 3.629)30) & $3.436(23)$ \\
\hline 1.10 & $3.111(23)$ & $3.305(19)$ & $3.405(21)$ & $3.477(29)$ & $3.414(22)$ & $3.358(23)$ & $3.547(19)$ & $3.633(26)$ & $3.491(17)$ \\
\hline \multicolumn{10}{|c|}{$\beta=4.05$} \\
\hline 0.60 & $2.600(43)$ & $2.946(29)$ & $3.107(32)$ & $3.199(46)$ & $3.115(34)$ & $3.034(38)$ & $3.336(23)$ & $3.400(29)$ & $3.287(22)$ \\
\hline 0.70 & $2.692(40)$ & $3.008(25)$ & $3.152(29)$ & $3.233(41)$ & $3.161(31)$ & $3.080(35)$ & $3.362(21)$ & $3.425(26)$ & $3.313(19)$ \\
\hline 0.80 & $2.788(46)$ & $3.074(32)$ & $3.200(34)$ & $3.269(49)$ & $3.209(36)$ & $3.127(40)$ & $3.391(25)$ & $3.451(32)$ & $3.340(24)$ \\
\hline 0.90 & $2.874(32)$ & $3.135(32)$ & $3.242(30)$ & $3.295(45)$ & $3.253(31)$ & $3.165(31)$ & $3.418(28)$ & $3.474(34)$ & $3.364(25)$ \\
\hline 1.00 & $2.984(33)$ & $3.205(32)$ & $3.298(30)$ & $3.348(45)$ & $3.308(31)$ & $3.230(31)$ & $3.448(29)$ & $3.504(34)$ & $3.397(25)$ \\
\hline 1.10 & $3.119(21)$ & $3.283(20)$ & $3.370(21)$ & $3.430(27)$ & $3.373(21)$ & $3.325(21)$ & $3.481(19)$ & $3.539(23)$ & $3.440(17)$ \\
\hline \multicolumn{10}{|c|}{ Continuum limit } \\
\hline 0.60 & $2.577(20)$ & $2.929(15)$ & $3.077(18)$ & $3.173(28)$ & $3.095(21)$ & $3.011(18)$ & $3.328(13)$ & $3.413(18)$ & $3.275(12)$ \\
\hline 0.70 & $2.676(21)$ & $3.003(15)$ & $3.179(17)$ & $3.258(28)$ & $3.198(17)$ & $3.101(20)$ & $3.383(13)$ & $3.477(18)$ & $3.316(13)$ \\
\hline 0.80 & $2.766(25)$ & $3.080(20)$ & $3.207(22)$ & $3.297(32)$ & $3.215(23)$ & $3.138(24)$ & $3.415(16)$ & $3.506(22)$ & $3.353(16)$ \\
\hline 0.90 & $2.878(20)$ & $3.149(18)$ & $3.267(19)$ & $3.359(29)$ & $3.275(20)$ & $3.193(20)$ & $3.456(16)$ & $3.552(21)$ & $3.387(15)$ \\
\hline 0.10 & $2.988(24)$ & $3.217(21)$ & $3.342(21)$ & $3.436(26)$ & $3.363(20)$ & $3.280(19)$ & $3.484(18)$ & $3.573(23)$ & $3.418(17)$ \\
\hline 1.10 & $3.116(15)$ & $3.295(14)$ & $3.387(15)$ & $3.452(20)$ & $3.392(15)$ & $3.340(15)$ & $3.514(13)$ & $3.580(17)$ & $3.465(12)$ \\
\hline
\end{tabular}

and $\Xi^{*}$ as well as for the $\Sigma$ at the smaller lattice spacing. In the case of the $\Xi$ we observe a nonzero splitting that decreases with the lattice spacing. If one interpolates the results at the two $\beta$ values to the same pion mass, as discussed in more detail in the next section, and makes a linear extrapolation in $a^{2}$ one finds that this splitting goes to zero in the continuum limit as expected. Whereas this confirms that this splitting is a cutoff effect, to perform a

TABLE XIII. The same as Table XII but for the decuplet baryons.

\begin{tabular}{|c|c|c|c|c|c|c|c|c|c|}
\hline$r_{0} m_{\pi}$ & $r_{0} m_{\Delta^{\mathrm{Av}}}$ & $r_{0} m_{\Sigma * \mathrm{Av}}$ & $r_{0} m_{\Sigma^{*+}}$ & $r_{0} m_{\Sigma^{* 0}}$ & $r_{0} m_{\Sigma^{*-}}$ & $r_{0} m_{\Xi * \mathrm{Av} .}$ & $r_{0} m_{\Xi^{* 0}}$ & $r_{0} m_{\Xi^{*-}}$ & $r_{0} m_{\Omega}$ \\
\hline \multicolumn{10}{|c|}{$\beta=3.9$} \\
\hline 0.60 & $3.312(51)$ & $3.565(51)$ & $3.558(49)$ & $3.564(56)$ & $3.660(53)$ & $3.671(27)$ & $3.689(31)$ & $3.662(28)$ & $4.131(26)$ \\
\hline 0.70 & $3.419(57)$ & $3.719(55)$ & $3.679(65)$ & $3.735(61)$ & $3.744(52)$ & $3.805(39)$ & $3.845(41)$ & $3.754(38)$ & $4.195(36)$ \\
\hline 0.80 & $3.631(48)$ & $3.850(50)$ & $3.824(62)$ & $3.856(55)$ & $3.853(55)$ & $3.856(46)$ & $3.925(45)$ & $3.812(49)$ & $4.252(37)$ \\
\hline 0.90 & $3.727(42)$ & $3.907(44)$ & $3.871(55)$ & $3.918(49)$ & $3.924(49)$ & $3.873(41)$ & $3.947(40)$ & $3.839(44)$ & $4.259(33)$ \\
\hline 1.00 & $3.761(47)$ & $3.911(46)$ & $3.839(55)$ & $3.954(58)$ & $3.981(52)$ & $3.862(45)$ & $3.922(43)$ & $3.840(44)$ & $4.240(35)$ \\
\hline 1.10 & $3.950(27)$ & $4.075(35)$ & $4.085(33)$ & $4.081(34)$ & $4.074(36)$ & $3.981(38)$ & $4.046(34)$ & $3.909(39)$ & $4.328(27)$ \\
\hline \multicolumn{10}{|c|}{$\beta=4.05$} \\
\hline 0.60 & $3.524(47)$ & $3.769(57)$ & $3.732(67)$ & $3.787(65)$ & $3.766(58)$ & $3.806(40)$ & $3.823(39)$ & $3.789(40)$ & $4.221(34)$ \\
\hline 0.70 & $3.581(43)$ & $3.789(52)$ & $3.752(62)$ & $3.803(57)$ & $3.795(53)$ & $3.817(37)$ & $3.832(36)$ & $3.802(37)$ & $4.202(30)$ \\
\hline 0.80 & $3.615(50)$ & $3.808(60)$ & $3.773(71)$ & $3.819(70)$ & $3.822(61)$ & $3.828(43)$ & $3.842(42)$ & $3.816(43)$ & $4.183(37)$ \\
\hline 0.90 & $3.617(40)$ & $3.804(45)$ & $3.768(49)$ & $3.834(76)$ & $3.829(46)$ & $3.829(32)$ & $3.834(34)$ & $3.825(33)$ & $4.141(36)$ \\
\hline 1.00 & $3.718(41)$ & $3.876(46)$ & $3.844(49)$ & $3.851(76)$ & $3.901(47)$ & $3.862(32)$ & $3.882(35)$ & $3.847(33)$ & $4.171(36)$ \\
\hline 1.10 & $3.922(40)$ & $4.028(39)$ & $4.007(45)$ & $3.868(49)$ & $4.042(38)$ & $3.931(29)$ & $3.987(33)$ & $3.885(29)$ & $4.277(33)$ \\
\hline \multicolumn{10}{|c|}{ Continuum limit } \\
\hline 0.60 & $3.439(35)$ & $3.656(38)$ & $3.619(40)$ & $3.660(42)$ & $3.709(39)$ & $3.712(22)$ & $3.741(24)$ & $3.704(23)$ & $4.164(21)$ \\
\hline 0.70 & $3.520(49)$ & $3.755(38)$ & $3.717(45)$ & $3.771(42)$ & $3.768(37)$ & $3.811(27)$ & $3.838(27)$ & $3.778(26)$ & $4.199(23)$ \\
\hline 0.80 & $3.623(35)$ & $3.833(38)$ & $3.802(47)$ & $3.841(43)$ & $3.839(41)$ & $3.841(31)$ & $3.880(30)$ & $3.814(32)$ & $4.217(26)$ \\
\hline 0.90 & $3.668(29)$ & $3.856(32)$ & $3.813(36)$ & $3.893(41)$ & $3.874(34)$ & $3.845(25)$ & $3.882(26)$ & $3.830(26)$ & $4.205(24)$ \\
\hline 1.00 & $3.735(30)$ & $3.893(32)$ & $3.842(37)$ & $3.916(46)$ & $3.937(35)$ & $3.862(26)$ & $3.898(27)$ & $3.845(26)$ & $4.207(25)$ \\
\hline 1.10 & $3.935(20)$ & $4.054(26)$ & $4.058(27)$ & $4.013(28)$ & $4.059(26)$ & $3.949(23)$ & $4.016(24)$ & $3.893(23)$ & $4.307(21)$ \\
\hline
\end{tabular}


proper analysis one would need results at an additional lattice spacing. For the current work we conclude that isospin splitting at these two lattice spacings is negligible for all baryons expect for the $\Xi$, where an isospin breaking of about $6 \%$ is observed that vanishes at a rate proportional to $a^{2}$.

\section{Continuum extrapolation}

In order to assess cutoff effects we use results at $\beta=3.9$ and $\beta=4.05$. The lattice results, expressed in units of the Sommer scale $r_{0}$, are interpolated to the same pion mass in units of $r_{0}$ at each $\beta$ value. We give the interpolated results at six values of $m_{\pi} r_{0}$ in Tables XII and XIII. Interpolating linearly or using leading order one-loop chiral perturbation theory gives values that are within error bars. Given the small size of the cutoff effects, a weighted average of the

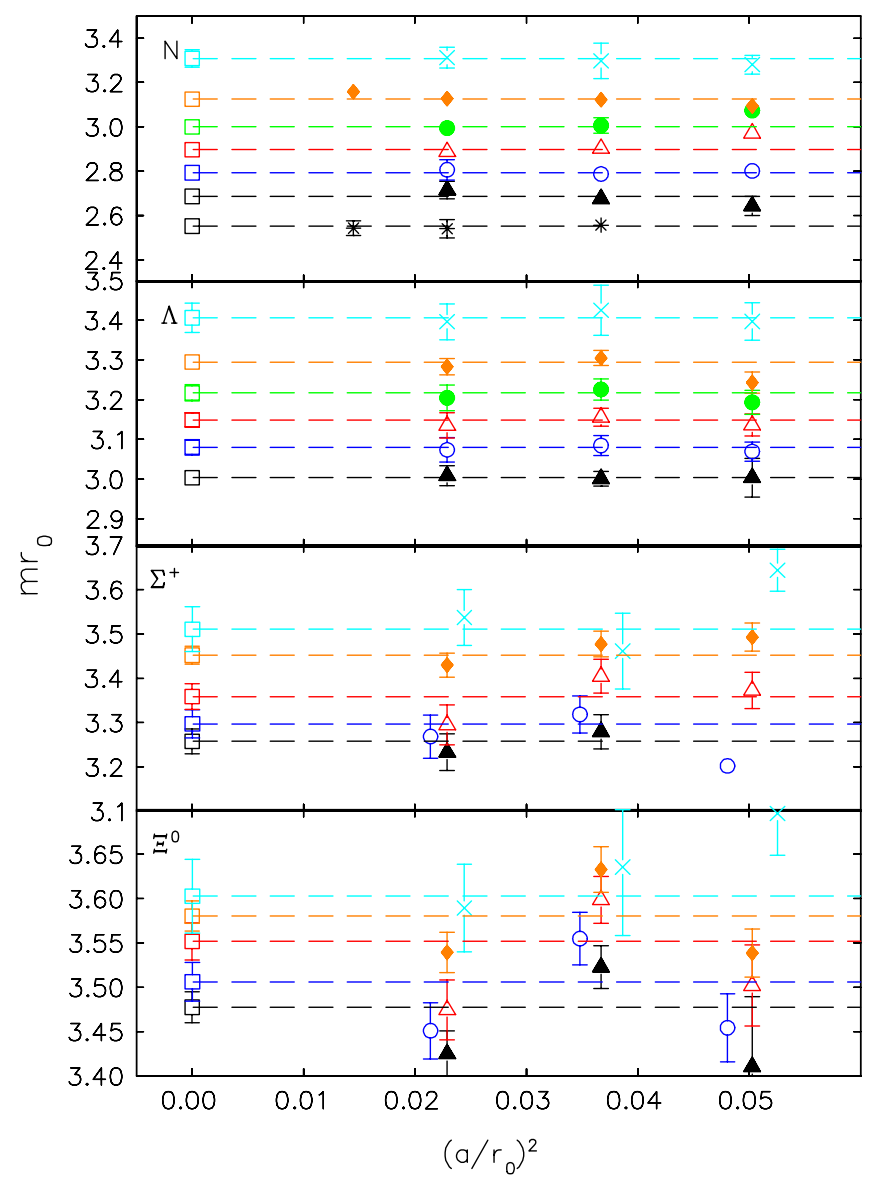

FIG. 11 (color online). Constant extrapolation to the continuum limit for the octet baryons. Stars are for $r_{0} m_{\pi}=0.615$, filled triangles for $r_{0} m_{\pi}=0.7$, open circles for $r_{0} m_{\pi}=0.8$, open triangles for $r_{0} m_{\pi}=0.9$, filled circles for $r_{0} m_{\pi}=1.0$, rhombii for $r_{0} m_{\pi}=1.1$, and crosses for $r_{0} m_{\pi}=1.25$. The open squares show the extracted continuum value. For the nucleon we also show results at $\beta=4$.2. For the $\Sigma^{+}$and $\Xi^{0}$ we omit the case $r_{0} m_{\pi}=1.0$ and shift results at $r_{0} m_{\pi}=1.25$ and $r_{0} m_{\pi}=$ 0.8 for clarity. baryon masses between these two $\beta$ values gives an estimate of the values in the continuum limit. It must be stressed that estimating the strange quark mass at $\beta=$ 4.05 using Eq. (10) may cause residual cutoff effects on the few percentage level that are not taken into account with the continuum extrapolation as performed here. The results obtained from the weighted averaging of data at $\beta=3.9$ and $\beta=4.05$ are listed in Tables XII and XIII and are plotted in Figs. 11 and 12. In the figures we also include results at $\beta=3$.8. If cutoff effects are small for all $\beta$ values then results at $\beta=3.8$ should fall onto the same line. As can be seen this is best fulfilled for the nucleon mass. Furthermore for the nucleon and the $\Delta$ we also show results at a smaller value of the lattice spacing corresponding to $\beta=4.2$. Essentially, the $a^{2}$ dependence of the nucleon and $\Delta$ mass as computed at $\beta=3.9,4.05$, and 4.2 is consistent with a constant behavior, verifying that for lattice spacings below $0.1 \mathrm{fm}$ cutoff effects are indeed small. For the $\Lambda$ mass results at $\beta=3.8,3.9$ and $\beta=4.05$ are consistent with a constant. This holds approximately also for the other baryons. Within the statistical errors one therefore concludes that for lattice spacings below $0.1 \mathrm{fm}$ cutoff effects are under control.

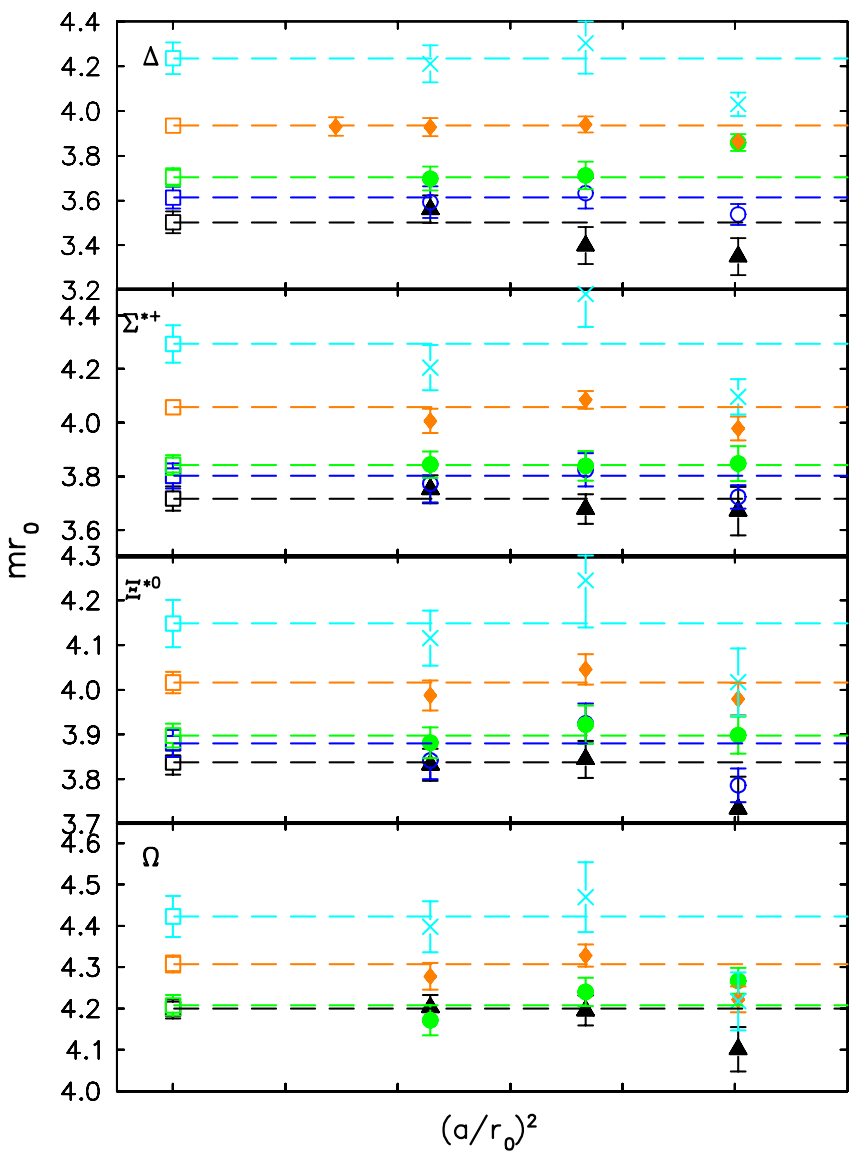

FIG. 12 (color online). Constant extrapolation to the continuum for the decuplet. The notation is the same as in Fig. 11. 


\section{Fixing the lattice spacing}

In order to convert to physical units we need to fix the lattice spacing. The value of the lattice spacings given in Table I was extracted using the pion decay constant. Equivalently, by extrapolating the pion decay constant to the physical point one finds $r_{0}=0.439(25) \mathrm{fm}$ [47], where the systematic error is added to the statistical one. Knowing $r_{0}$ and the ratio $r_{0} / a$ one can determine the lattice spacing.

Alternatively, the nucleon mass can be used to set the scale and this determination seems natural if one is interested in the study of the baryon spectrum. Our data at $\beta=$ 3.9 and $\beta=4.05$ do not show significant lattice spacing effects. Therefore we can make a combined fit using continuum chiral perturbation theory to determine the value of $r_{0}^{N}$, where the superscript $N$ denotes using the nucleon mass. Chiral corrections to the nucleon mass are known to $\mathcal{O}\left(p^{4}\right)$ within several expansion schemes. We use the same schemes as in Refs. [4,48]. The fits are shown in Fig. 13. Using the $\mathcal{O}\left(p^{3}\right)$ result, which is well established, to extrapolate to the physical pion mass we obtain $r_{0}^{N}=$ $0.465(6) \mathrm{fm}$. If we instead use the data at $\beta=3.9$ and $\beta=$ 4.05 to perform the continuum limit as discussed in the previous section and then fit, we find $r_{0}^{N}=0.471 \pm$ 0.006 (stat) \pm 0.015 (syst) fm. The systematic error is due to the interpolation to a fixed value of $m_{\pi} r_{0}$ and it is estimated by comparing the value of $r_{0}$ obtained when linear interpolation is used to the one obtained using $\mathcal{O}\left(p^{3}\right)$. Furthermore, we take the difference in the value of $r_{0}$ obtained using continuous results and the value found by fitting the lattice data at $\beta=3.9$ and $\beta=4.05$ to be the systematic error due to cutoff effects. We therefore take $r_{0}^{N}=0.465(6)(14) \mathrm{fm}$, which is in agreement with $r_{0}$ extracted from the value of the pion decay constant, $f_{\pi}$. Using the values of $r_{0} / a=5.22(2)$ and $r_{0} / a=6.61(3)$ at $\beta=3.9$ and $\beta=4.05$, we find for the lattice spacings $a_{\beta=3.9}=0.089(4) \mathrm{fm}$ and $a_{\beta=4.05}=0.070(3) \mathrm{fm}$. These

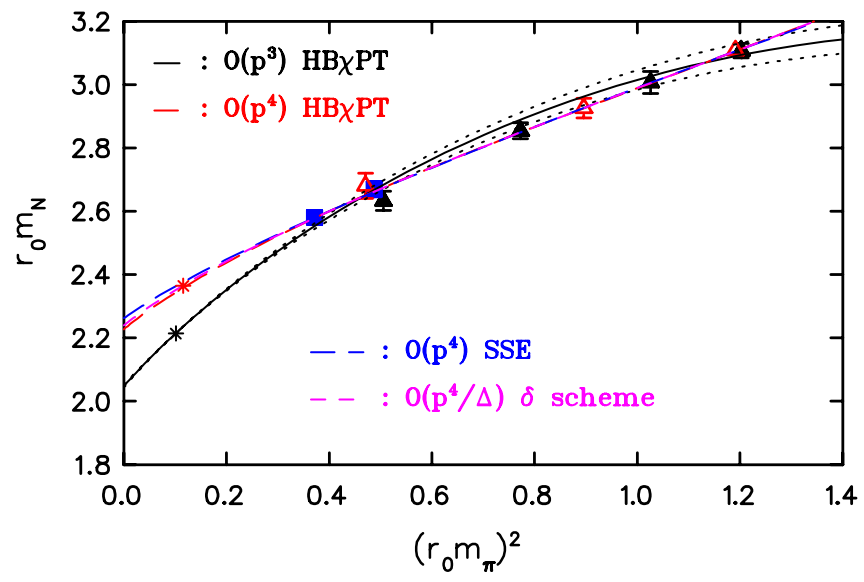

FIG. 13 (color online). Determination of $r_{0}^{N}$ with a simultaneous fit to the lattice data at $\beta=3.9$ and $\beta=4.05$. The asterisks denote the physical point determined by the value of $r_{0}^{N}$ by using $\mathcal{O}\left(p^{3}\right)$ and $\mathcal{O}\left(p^{4}\right) \chi \mathrm{PT}$ as described in Ref. [4]. values for the lattice spacing extracted using the nucleon mass at the physical point are in agreement with those determined from the pion decay constant. This constitutes a nice consistency check of our lattice formulation. In what follows we will use the lattice spacing extracted from the nucleon mass using $\mathcal{O}\left(p^{3}\right)$ heavy baryon chiral perturbation theory to convert the rest of the masses into physical units.

\section{CHIRAL EXTRAPOLATION}

Given that the cutoff effects are almost negligible in our simulations, we apply continuum chiral perturbation theory to extrapolate lattice results at $\beta=3.9$ and $\beta=4.05$ to the physical pion mass. In particular, we will use SU(2) $\chi$ PT [49] for two reasons: the first being that our simulations are done for two mass-degenerate dynamical quarks and the second because it was shown that $\mathrm{SU}(3) \chi \mathrm{PT}$ fails to describe lattice data [46]. We would like to stress however that the issue of the applicability of SU(3) $\chi \mathrm{PT}$ is not entirely settled and e.g. SU(3) fits to lattice results using staggered fermions were claimed to produce reasonable fits [50,51].

The baryon masses to leading order (LO) SU(2) heavy baryon chiral perturbation $(\mathrm{HB} \chi \mathrm{PT})$ are given by

$$
m_{X}^{\mathrm{LO}}\left(m_{\pi}\right)=m_{X}^{(0)}-4 c_{X}^{(1)} m_{\pi}^{2}
$$

with two fit parameters, the baryon mass in the chiral limit $m_{X}^{(0)}$, and $c_{X}^{(1)}$, which gives the leading contribution to the $\sigma_{X}$ term.

The leading one-loop results for the nucleon and the $\Delta$ in $\mathrm{HB} \chi \mathrm{PT}$ were first derived in Ref. [52] and successful fits to lattice data on the nucleon and $\Delta$ were discussed in our previous study [4]. A natural generalization of the $\mathcal{O}\left(p^{3}\right)$ results for the nucleon and $\Delta$ to the rest of the octet and decuplet baryons $[53,54]$ is given by

$$
\begin{aligned}
& m_{N}\left(m_{\pi}\right)=m_{N}^{(0)}-4 c_{N}^{(1)} m_{\pi}^{2}-\frac{3 g_{A}^{2}}{16 \pi f_{\pi}^{2}} m_{\pi}^{3}, \\
& m_{\Lambda}\left(m_{\pi}\right)=m_{\Lambda}^{(0)}-4 c_{\Lambda}^{(1)} m_{\pi}^{2}-\frac{g_{\Lambda \Sigma}^{2}}{16 \pi f_{\pi}^{2}} m_{\pi}^{3}, \\
& m_{\Sigma}\left(m_{\pi}\right)=m_{\Sigma}^{(0)}-4 c_{\Sigma}^{(1)} m_{\pi}^{2}-\frac{2 g_{\Sigma \Sigma}^{2}+g_{\Lambda \Sigma}^{2} / 3}{16 \pi f_{\pi}^{2}} m_{\pi}^{3}, \\
& m_{\Xi}\left(m_{\pi}\right)=m_{\Xi}^{(0)}-4 c_{\Xi}^{(1)} m_{\pi}^{2}-\frac{3 g_{\Xi \Xi}^{2}}{16 \pi f_{\pi}^{2}} m_{\pi}^{3},
\end{aligned}
$$

for the octet baryons and 


$$
\begin{aligned}
& m_{\Delta}\left(m_{\pi}\right)=m_{\Delta}^{(0)}-4 c_{\Delta}^{(1)} m_{\pi}^{2}-\frac{25}{27} \frac{g_{\Delta \Delta}^{2}}{16 \pi f_{\pi}^{2}} m_{\pi}^{3}, \\
& m_{\Sigma^{*}}\left(m_{\pi}\right)=m_{\Sigma^{*}}^{(0)}-4 c_{\Sigma^{*}}^{(1)} m_{\pi}^{2}-\frac{10}{9} \frac{g_{\Sigma^{*} \Sigma^{*}}^{2}}{16 \pi f_{\pi}^{2}} m_{\pi}^{3}, \\
& m_{\Xi^{*}}\left(m_{\pi}\right)=m_{\Xi^{*}}^{(0)}-4 c_{\Xi^{*}}^{(1)} m_{\pi}^{2}-\frac{5}{3} \frac{g_{\Xi^{*} \Xi^{*}}^{2}}{16 \pi f_{\pi}^{2}} m_{\pi}^{3}, \\
& m_{\Omega}\left(m_{\pi}\right)=m_{\Omega}^{(0)}-4 c_{\Omega}^{(1)} m_{\pi}^{2},
\end{aligned}
$$

for the decuplet baryons.
In addition we consider a cubic term of the following form:

$$
m_{X}\left(m_{\pi}\right)=m_{X}^{(0)}-4 c_{X}^{(1)} m_{\pi}^{2}+c_{X}^{(2)} m_{\pi}^{3}
$$

treating $c_{X}^{(2)}$ as an additional fit parameter.

The next-to-leading order (NLO) SU(2) $\chi \mathrm{PT}$ results [49] for the octet are given by

$$
\begin{aligned}
& m_{N}^{\mathrm{NLO}}\left(m_{\pi}\right)=m_{N}^{\mathrm{LO}}\left(m_{\pi}\right)-\frac{3 g_{A}^{2}}{16 \pi f_{\pi}^{2}} m_{\pi}^{3}-\frac{8 g_{N \Delta}^{2}}{3\left(4 \pi f_{\pi}\right)^{2}} \mathcal{F}\left(m_{\pi}, \Delta_{N \Delta}, \lambda\right), \\
& m_{\Lambda}^{\mathrm{NLO}}\left(m_{\pi}\right)=m_{\Lambda}^{\mathrm{LO}}\left(m_{\pi}\right)-\frac{g_{\Lambda \Sigma}^{2}}{\left(4 \pi f_{\pi}\right)^{2}} \mathcal{F}\left(m_{\pi}, \Delta_{\Lambda \Sigma}, \lambda\right)-\frac{4 g_{\Lambda \Sigma^{*}}^{2}}{\left(4 \pi f_{\pi}\right)^{2}} \mathcal{F}\left(m_{\pi}, \Delta_{\Lambda \Sigma^{*}}, \lambda\right), \\
& m_{\Sigma}^{\mathrm{NLO}}\left(m_{\pi}\right)=m_{\Sigma}^{\mathrm{LO}}\left(m_{\pi}\right)-\frac{2 g_{\Sigma \Sigma}^{2}}{16 \pi f_{\pi}^{2}} m_{\pi}^{3}-\frac{g_{\Lambda \Sigma}^{2}}{3\left(4 \pi f_{\pi}\right)^{2}} \mathcal{F}\left(m_{\pi},-\Delta_{\Lambda \Sigma}, \lambda\right)-\frac{4 g_{\Lambda \Sigma^{*}}^{2}}{3\left(4 \pi f_{\pi}\right)^{2}} \mathcal{F}\left(m_{\pi}, \Delta_{\Sigma \Sigma^{*}}, \lambda\right), \\
& m_{\Xi}^{\mathrm{NLO}}\left(m_{\pi}\right)=m_{\Xi}^{\mathrm{LO}}\left(m_{\pi}\right)-\frac{3 g_{\Xi \Xi}^{2}}{16 \pi f_{\pi}^{2}} m_{\pi}^{3}-\frac{2 g_{\Xi *}^{2} \Xi}{\left(4 \pi f_{\pi}\right)^{2}} \mathcal{F}\left(m_{\pi}, \Delta_{\Xi \Xi^{*}}, \lambda\right),
\end{aligned}
$$

and for the decuplet baryons:

$$
\begin{aligned}
& m_{\Delta}^{\mathrm{NLO}}\left(m_{\pi}\right)=m_{\Delta}^{\mathrm{LO}}\left(m_{\pi}\right)-\frac{25}{27} \frac{g_{\Delta \Delta}^{2}}{16 \pi f_{\pi}^{2}} m_{\pi}^{3}-\frac{2 g_{\Delta N}^{2}}{3\left(4 \pi f_{\pi}\right)^{2}} \mathcal{F}\left(m_{\pi},-\Delta_{N \Delta}, \lambda\right), \\
& m_{\Sigma^{*}}^{\mathrm{NLO}}\left(m_{\pi}\right)=m_{\Sigma^{*}}^{\mathrm{LO}}\left(m_{\pi}\right)-\frac{10}{9} \frac{g_{\Sigma^{*} \Sigma^{*}}^{2}}{16 \pi f_{\pi}^{2}} m_{\pi}^{3}-\frac{2}{3\left(4 \pi f_{\pi}\right)^{2}}\left[g_{\Sigma^{*} \Sigma}^{2} \mathcal{F}\left(m_{\pi},-\Delta_{\Sigma \Sigma^{*}, \lambda}\right)+g_{\Lambda \Sigma^{*}}^{2} \mathcal{F}\left(m_{\pi},-\Delta_{\Lambda \Sigma^{*}, \lambda}\right)\right] \\
& m_{\Xi^{*}}^{\mathrm{NLO}}\left(m_{\pi}\right)=m_{\Xi^{*}}^{\mathrm{LO}}\left(m_{\pi}\right)-\frac{5}{3} \frac{g_{\Xi^{*} \Xi^{*}}^{2}}{16 \pi f_{\pi}^{2}} m_{\pi}^{3}-\frac{g_{\Xi^{*} \Xi}^{2}}{\left(4 \pi f_{\pi}\right)^{2}} \mathcal{F}\left(m_{\pi},-\Delta_{\Xi \Xi^{*}, \lambda}\right), \\
& m_{\Omega}^{\mathrm{NLO}}\left(m_{\pi}\right)=m_{\Omega}^{\mathrm{LO}}\left(m_{\pi}\right),
\end{aligned}
$$

with the nonanalytic function [55]

$$
\mathcal{F}(m, \Delta, \lambda)=\left(m^{2}-\Delta^{2}\right) \sqrt{\Delta^{2}-m^{2}+i \epsilon} \log \left(\frac{\Delta-\sqrt{\Delta^{2}-m^{2}+i \epsilon}}{\Delta+\sqrt{\Delta^{2}-m^{2}+i \epsilon}}\right)-\frac{3}{2} \Delta m^{2} \log \left(\frac{m^{2}}{\lambda^{2}}\right)-\Delta^{3} \log \left(\frac{4 \Delta^{2}}{m^{2}}\right)
$$

depending on the threshold parameter $\Delta_{X Y}=m_{Y}^{(0)}-m_{X}^{(0)}$ and on the scale $\lambda$ of chiral perturbation theory, fixed to $\lambda=$ $1 \mathrm{GeV}$. For $\Delta>0$ the real part of the function $\mathcal{F}(m, \Delta, \lambda)$ has the property

$$
\mathcal{F}(m,-\Delta, \lambda)= \begin{cases}-\mathcal{F}(m, \Delta, \lambda) & m<\Delta \\ -\mathcal{F}(m, \Delta, \lambda)+2 \pi\left(m^{2}-\Delta^{2}\right)^{3 / 2} & m>\Delta\end{cases}
$$

which corrects a typo in the sign of the second term in Ref. [8]. In our fits, the nucleon axial charge $g_{A}$ and pion decay constant $f_{\pi}$ are fixed to their experimental values (we use the convention such that $f_{\pi}=130.70 \mathrm{MeV}$ ). The remaining pion-baryon axial coupling constants are taken from $\mathrm{SU}(3)$ relations [49]:

$$
\begin{array}{lllll}
\text { octet: } & g_{A}=D+F, & g_{\Sigma \Sigma}=2 F, & g_{\Xi \Xi}=D-F, & g_{\Lambda \Sigma}=2 D, \\
\text { decuplet: } & g_{\Delta \Delta}=\mathcal{H}, & g_{\Sigma^{*} \Sigma^{*}}=\frac{2}{3} \mathcal{H}, & g_{\Xi^{*} \Xi^{*}}=\frac{1}{3} \mathcal{H}, & \\
\text { transition: } & g_{\Delta N}=\mathcal{C}, & g_{\Sigma^{*} \Sigma}=\frac{1}{\sqrt{3}} \mathcal{C}, & g_{\Xi^{*} \Xi}=\frac{1}{\sqrt{3}} \mathcal{C}, & g_{\Lambda \Sigma^{*}}=-\frac{1}{\sqrt{2}} \mathcal{C} .
\end{array}
$$


As can be seen, in the octet case, and once $g_{A}$ is fixed, the axial coupling constants depend on the single parameter written as $\alpha=\frac{D}{D+F}$. Its value is poorly known. It can be taken either from the quark model $(\alpha=3 / 5)$, from the phenomenology of semileptonic decays, or from hyperonnucleon scattering. We take $2 D=1.47$ or $\alpha=0.58$ as given in Ref. [49]. The decuplet coupling constants depend on a single parameter for which we again take the value $\mathcal{H}=2.2$ from Ref. [49]. This value is not far from that predicted by $\mathrm{SU}(6)$ symmetry, $\mathcal{H}=\frac{9}{5} g_{A}=2.29$, used in our previous work [4] resulting in the same cubic term for the nucleon and $\Delta$. For fixing the octet-decuplet transition couplings we take the value $\mathcal{C}=1.48$ from Ref. [55].

With the coupling constants fixed in this way, the LO, the one-loop as well as the NLO fits are left with the two independent fit parameters $m_{X}^{(0)}$ and $c_{X}^{(1)}$. All mass parameters $m_{X}^{(0)}$ are treated independently unlike what is done in Ref. [49], where a universal mass parameter was used for all barons with the same strangeness.

It is worth commenting on the absence of an explicit cubic term in the expressions for the $\Lambda$ and $\Omega$ masses given by Eqs. (26) and (27), which is of a different origin in each case. In the case of $\Omega$, it follows from the absence of light valence quarks. The absence of a cubic term in the NLO expression of $\Lambda$ is a consequence of treating $m_{\pi} \sim M_{\Sigma}-$ $M_{\Lambda}$ in $\chi \mathrm{PT}$ expansions, which however is not satisfied either by the experimental values nor by the lattice results, but rather indicate $M_{\Sigma}-M_{\Lambda} \ll m_{\pi}$. It is thus justified to consider the $\Delta \rightarrow 0$ limit of the nonanalytic function (28)

$$
\mathcal{F}\left(m_{\pi}, \Delta \rightarrow 0, \lambda\right)=\pi m_{\pi}^{3},
$$

which generates a cubic term for the $\Lambda$ and slightly modifies the one for $\Sigma$. The corresponding expressions are given by

$$
\begin{aligned}
& m_{\Lambda}\left(m_{\pi}\right)=m_{\Lambda}^{(0)}-4 c_{\Lambda}^{(1)} m_{\pi}^{2}-\frac{g_{\Lambda \Sigma}^{2}}{16 \pi f_{\pi}^{2}} m_{\pi}^{3}, \\
& m_{\Sigma}\left(m_{\pi}\right)=m_{\Sigma}^{(0)}-4 c_{\Sigma}^{(1)} m_{\pi}^{2}-\frac{2 g_{\Sigma \Sigma}^{2}+g_{\Lambda \Sigma}^{2} / 3}{16 \pi f_{\pi}^{2}} m_{\pi}^{3},
\end{aligned}
$$

in agreement with the results of Eq. (23).

The expressions for the strange baryon masses to NNLO in $\chi$ PT given in Ref. [49] involve in general more unknown low energy constants and only if we perform a constrained fit do we have enough data to extract them. We found however no real advantage in using constrained fits, which generally gave larger $\chi /$ d.o.f. and did not improve the prediction of the mass at the physical point as compared to the unconstrained fits. For the nucleon, $\Delta$ and $\Omega$ masses, unconstrained fits can still be performed with four fit parameters [8], namely, $m^{(0)}, c^{(1)}, \alpha$, and $\beta$ appearing in the expressions of NNLO $\chi$ PT given below:

$$
\begin{aligned}
& m_{N}^{\mathrm{NNLO}}\left(m_{\pi}\right)=m_{N}^{\mathrm{NLO}}\left(m_{\pi}\right)+m_{\pi}^{4}\left[\beta_{N}+\frac{16 g_{\Delta N}^{2} c_{N}^{(1)}}{\left(4 \pi f_{\pi}\right)^{2}}-\frac{9 g_{\Delta N}^{2}}{4 m_{N}^{(0)}\left(4 \pi f_{\pi}\right)^{2}}-\frac{45 g_{A}^{2}}{324 m_{N}^{(0)}\left(4 \pi f_{\pi}\right)^{2}}\right]+\frac{16 g_{\Delta N}^{2} c_{N}^{(1)}}{\left(4 \pi f_{\pi}\right)^{2}} m_{\pi}^{2} \mathcal{J}\left(m_{\pi}, \Delta, \lambda\right) \\
& +\frac{m_{\pi}^{4}}{\left(4 \pi f_{\pi}\right)^{2}} \log \left(\frac{m_{\pi}^{2}}{\lambda^{2}}\right)\left[12 c_{N}^{(1)}-\frac{3 \alpha_{N}}{4 \pi f_{\pi}}-\frac{27 g_{A}^{2}}{16 m_{N}^{(0)}}-\frac{5 g_{\Delta N}}{2 m_{N}(0)}\right] \\
& m_{\Delta}^{\mathrm{NNLO}}\left(m_{\pi}\right)=m_{\Delta}^{\mathrm{NLO}}\left(m_{\pi}\right)+\frac{12 c_{\Delta}^{(1)}}{\left(4 \pi f_{\pi}\right)^{2}} m_{\pi}^{4} \log \left(\frac{m_{\pi}^{2}}{\lambda^{2}}\right)-\frac{25 g_{\Delta \Delta}^{2}}{48\left(m_{\Delta}^{(0)}+\Delta_{\Delta N}\right)\left(4 \pi f_{\pi}\right)^{2}} m_{\pi}^{4}\left(\log \left(\frac{m_{\pi}^{2}}{\lambda^{2}}\right)+\frac{19}{10}\right) \\
& -\frac{5 g_{\Delta N}^{2}}{8\left(m_{\Delta}^{(0)}+\Delta_{\Delta N}\right)\left(4 \pi f_{\pi}\right)^{2}} m_{\pi}^{4}\left(\log \left(\frac{m_{\pi}^{2}}{\lambda^{2}}\right)-\frac{1}{10}\right)+\frac{4 c_{\Delta}^{(1)} g_{\Delta N}^{2}}{\left(4 \pi f_{\pi}\right)^{2}} m_{\pi}^{2} \mathcal{J}\left(m_{\pi},-\Delta_{\Delta N}, \lambda\right)+\beta_{\Delta} m_{\pi}^{4} \\
& +\frac{\alpha_{\Delta}}{\left(4 \pi f_{\pi}\right)^{3}} m_{\pi}^{4} \log \left(\frac{m_{\pi}^{2}}{\lambda^{2}}\right) \\
& m_{\Omega}^{\mathrm{NNLO}}\left(m_{\pi}\right)=m_{\Omega}^{\mathrm{NLO}}\left(m_{\pi}\right)+\frac{m_{\pi}^{4}}{\left(4 \pi f_{\pi}\right)^{3}}\left[\alpha_{\Omega} \log \left(\frac{m_{\pi}^{2}}{\lambda^{2}}\right)+\beta_{\Omega}\right],
\end{aligned}
$$

where

$$
\mathcal{J}(m, \Delta, \lambda)=m^{4} \log \left(\frac{m^{2}}{\lambda^{2}}\right)+2 \Delta \sqrt{\Delta^{2}-m^{2}+i \epsilon} \log \left(\frac{\Delta-\sqrt{\Delta^{2}-m^{2}+i \epsilon}}{\Delta+\sqrt{\Delta^{2}-m^{2}+i \epsilon}}\right)+2 \Delta^{2} \log \left(\frac{4 \Delta^{2}}{m^{2}}\right),
$$

and the real part of $\mathcal{J}$ satisfies

$$
\mathcal{J}(m,-\Delta, \lambda)= \begin{cases}\mathcal{J}(m, \Delta, \lambda) & m<\Delta \\ \mathcal{J}(m, \Delta, \lambda)-2 \pi \Delta\left(m^{2}-\Delta^{2}\right)^{1 / 2} & m>\Delta\end{cases}
$$




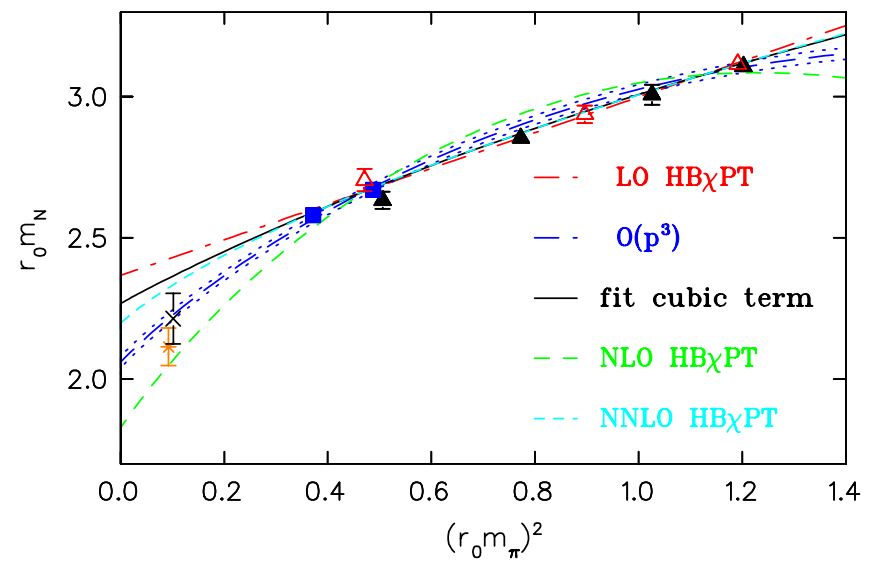

FIG. 14 (color online). Chiral extrapolation of the nucleon mass in units of $r_{0}$. Filled triangles and squares are results at $\beta=3.9$ on $24^{3} \times 48$ and $32^{3} \times 64$ lattice sizes, respectively. Open triangles are results at $\beta=4.05$. We show chiral extrapolations linear in $m_{\pi}^{2}$ as in Eq. (22), to $\mathcal{O}\left(p^{3}\right)$ as in Eq. (23), fit including a cubic term as in Eq. (25), NLO and NNLO in SU(2) chiral perturbation theory as in Eqs. (26) and (32), respectively. We include an error band only for the $\mathcal{O}\left(p^{3}\right)$ fit for clarity. The physical point shown by the asterisk uses the value of $r_{0}$ extracted from $f_{\pi}$, whereas the cross uses $r_{0}^{N}$ determined from the nucleon mass.

Using the above Ansätze the chiral extrapolations of lattice results at $\beta=3.9$ and $\beta=4.05$ given in Tables V, VI, and VII are performed. In Fig. 14 we show the fits for the nucleon in units of $r_{0}$. The nucleon mass at the physical point has been expressed in units of $r_{0}$ using the value determined from the nucleon mass as well as from the pion decay constant. As can be seen these values are consistent. The $\mathcal{O}\left(p^{3}\right)$ being the one used to determine the scale passes through the physical point. The other curves show the dependence on the chiral Ansatz used. It comes as no surprise that the NLO result does badly for the nucleon underestimating the mass at the physical point, whereas the NNLO fits overcorrect and yield a higher mass. Lattice results at $\beta=3.9$ and 4.05 expressed in units of $r_{0}$ fall on a universal curve confirming that finite cutoff effects are small. Therefore we corroborate the conclusion that we can use continuum chiral perturbation theory to extrapolate lattice results at $\beta=3.9$ and $\beta=4.05$. For the chiral extrapolation of the other baryons we use the scale determined from the nucleon mass to convert to physical units.

We show in Fig. 15 the chiral extrapolation for the $\Delta$ mass and in Figs. 16, 18, and 20 the chiral extrapolations for the octet baryon masses. In Figs. 17, 19, and 21 we show the corresponding fits for the decuplet baryons. We emphasize that the physical point is not included in these fits.

The LO expression describes well the lattice results but leads to extrapolated values inconsistent with the experi-

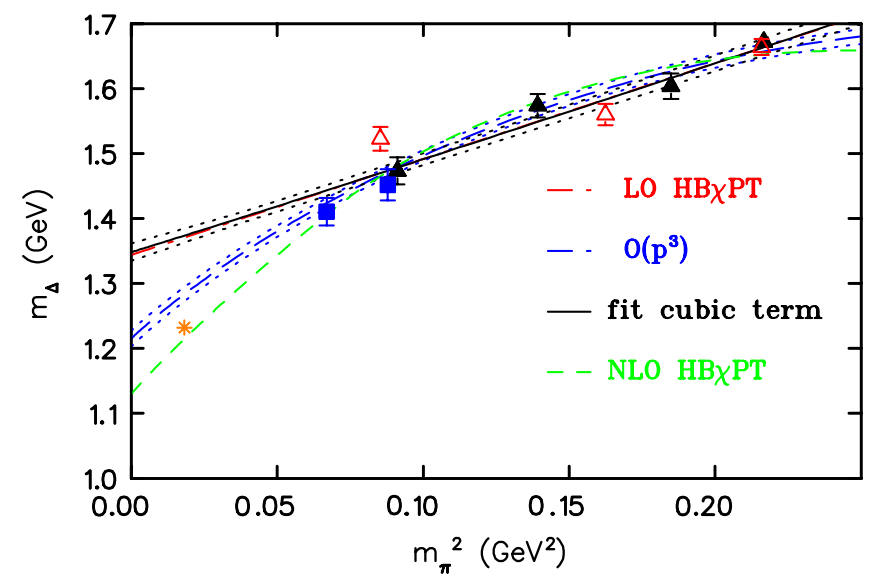

FIG. 15 (color online). Chiral extrapolation of the $\Delta$ mass. The notation is the same as that in Fig. 14 but in physical units. Here we also include an error band for the cubic fit.

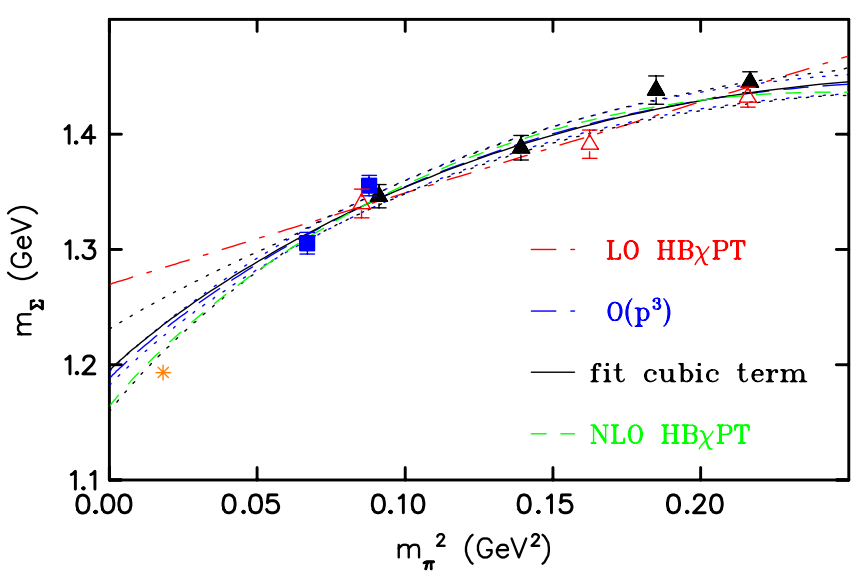

FIG. 16 (color online). Chiral extrapolation of the $\Sigma$ mass in physical units. We show chiral extrapolations linear in $m_{\pi}^{2}$, using Eq. (25) with a cubic term, $\mathcal{O}\left(p^{3}\right)$ given in Eq. (23) and NLO $\mathrm{SU}(2)$ chiral perturbation theory given in Eq. (26). The rest of the notation is the same as that in Fig. 15.

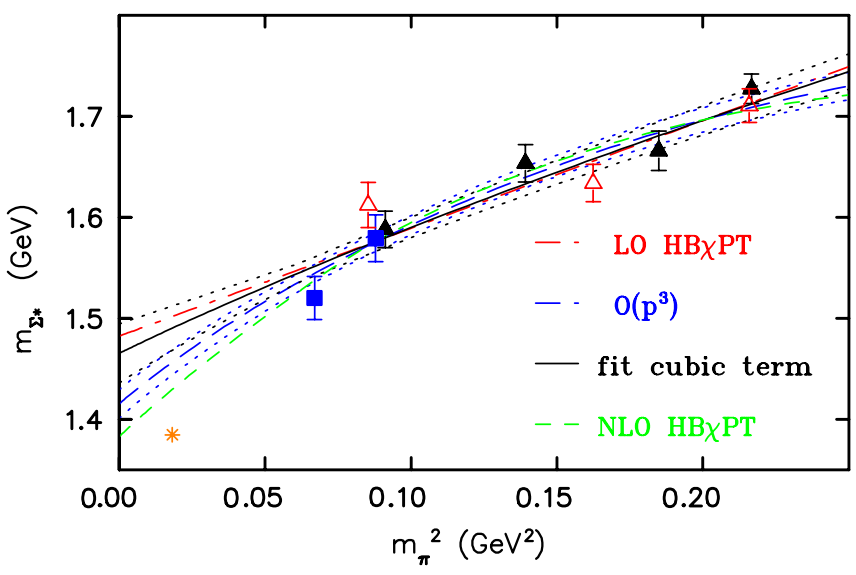

FIG. 17 (color online). Chiral extrapolation of the $\Sigma^{*}$ mass. The notation is the same as that in Fig. 16. 


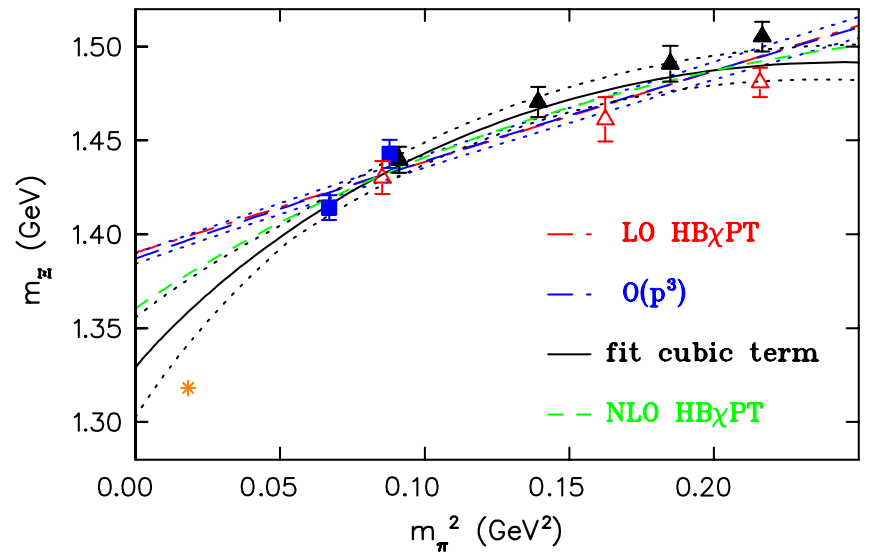

FIG. 18 (color online). Chiral extrapolation of the $\Xi$ mass. The notation is the same as that in Fig. 16.

mental point. The $\mathcal{O}\left(p^{3}\right) \mathrm{HB} \chi \mathrm{PT}$ expansion given in Eqs. (23) and (24) with two fit parameters $m^{(0)}$ and $c^{(1)}$ provides a good description of lattice data and the results extrapolated to the physical point are, in general, in agreement with experiments. The NLO leads to a clear improvement in the case of the $\Lambda$ and $\Xi$ masses, whereas for the rest of the baryons the improvement is marginal. Apart from the preceding remarks, there is no clear advantage in using higher order fits, especially NNLO, which even turns out to be numerically unstable for the case of the $\Delta$ and $\Omega$ masses. Therefore our main conclusion is that the $\mathcal{O}\left(p^{3}\right)$ $\mathrm{HB} \chi \mathrm{PT}$ provides a reasonable description for the nucleon and $\Delta$ masses, whereas for the strange baryon masses NLO $\mathrm{SU}(2)$ is preferable, yielding values at the physical point that are in agreement with experiment.

We use the relation $m_{\pi}^{2} \sim \mu$ to evaluate the nucleon $\sigma$ term by computing $m_{\pi}^{2} \frac{d M_{N}}{d m_{\pi}^{2}}$. Using our $\mathcal{O}\left(p^{3}\right)$ fit we find $\sigma_{N}=64.2(9) \mathrm{MeV}$ in agreement with the value given in Ref. [4]. Applying the same relation to the other baryons we can evaluate the corresponding $\sigma$ term for them. We list in Table XIV the values we obtain using the nucleon mass

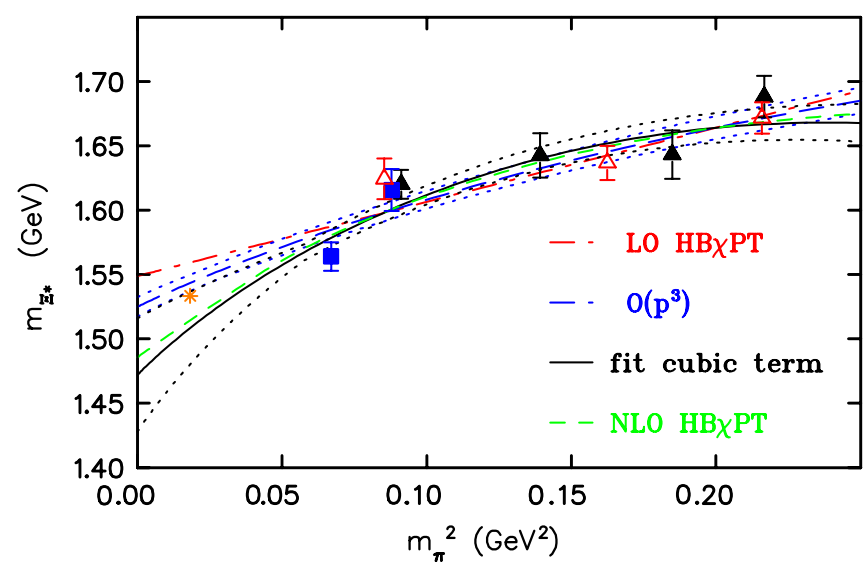

FIG. 19 (color online). Chiral extrapolation of the $\Xi^{*}$ mass. The notation is the same as that in Fig. 16.

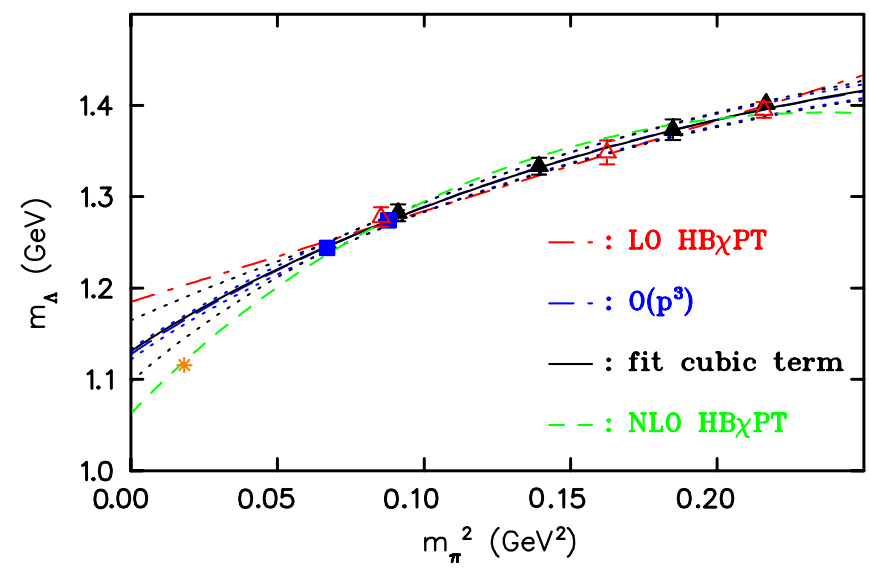

FIG. 20 (color online). Chiral extrapolation of the $\Lambda$ mass. The notation is the same as that in Fig. 16.

to set the scale. As can be seen, the value extracted depends on the fitting Ansatz. In the most interesting case of the nucleon the result of $\mathcal{O}\left(p^{3}\right)$ changes by 2 standard deviations if the coefficient of the cubic term in $m_{\pi}$ is fitted. In the case of the $\Lambda$ fitting the cubic term gives the same value as that obtained when using $\mathcal{O}\left(p^{3}\right)$, which agrees with the value found in the case of the nucleon. This is another indication of the argument presented above in favor of the presence of a cubic term in $m_{\pi}$ of comparable size as that of the nucleon. The main conclusion drawn from allowing the coefficient of the cubic term to be determined from the data is that the value of the $\sigma$ term for all baryons except the $\Omega$ is comparable within error to the value of the nucleon $\sigma$ term. Comparing to the results of NLO we can see that for the nucleon this fit produces too much curvature as already observed for instance in Ref. [8]. For the other baryons a reasonable value is obtained depending on the quality of the fits.

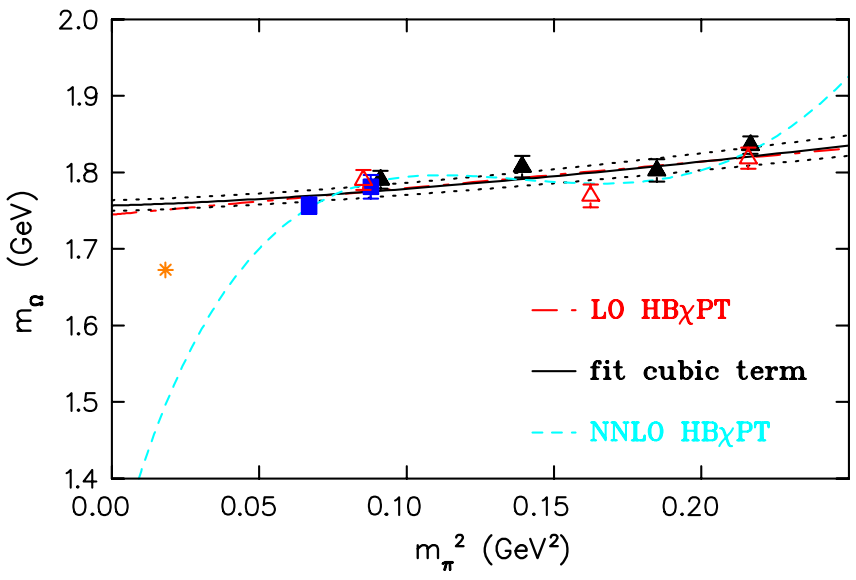

FIG. 21 (color online). Chiral extrapolation of the $\Omega$ mass. We show chiral extrapolations linear in $m_{\pi}^{2}$, using Eq. (25), and NNLO in SU(2) chiral perturbation theory given in Eq. (34). The rest of the notation is the same as that in Fig. 14. 
TABLE XIV. $\sigma$ term in MeV using the fit parameters determined from $\mathcal{O}\left(p^{3}\right) \chi \mathrm{PT}$, using a cubic fit Eq. (25) and NLO. We used the scale from the nucleon mass to convert to physical units.

\begin{tabular}{|c|c|c|c|c|c|c|c|c|}
\hline & $\sigma_{N}$ & $\sigma_{\Lambda}$ & $\sigma_{\sum^{\mathrm{Av}}}$ & $\sigma_{\Xi_{\mathrm{Av} .}}$ & $\sigma_{\Delta^{\mathrm{Av}}}$ & $\sigma_{\Sigma^{* \mathrm{Av}}}$ & $\sigma_{\Xi^{* \mathrm{Av} .}}$ & $\sigma_{\Omega}$ \\
\hline $\mathcal{O}\left(p^{3}\right)$ & $64.2(8)$ & 34.7(9) & $37.1(8)$ & $9.7(1.1)$ & $62.2(1.1)$ & $38.0(1.7)$ & $17.3(1.3)$ & $6.3(1.3)$ \\
\hline Fit with cubic term & $33.4(6.9)$ & $33.7(9.8)$ & $35.6(10.6)$ & $26.3(7.7)$ & $25.7(2.5)$ & $24.2(6.4)$ & $32.4(13.4)$ & $2.9(1.4)$ \\
\hline NLO & $92.5(7)$ & $52.8(8)$ & $43.3(9)$ & $17.2(1.0)$ & $79.5(1.0)$ & $44.1(1.7)$ & $27.9(1.3)$ & $6.3(1.3)$ \\
\hline
\end{tabular}

\section{COMPARISON WITH OTHER LATTICE RESULTS AND WITH EXPERIMENT}

In this section we show a comparison of recent lattice results on the baryon masses from various collaborations in Figs. 22 and 23. For our results we use the lattice spacing

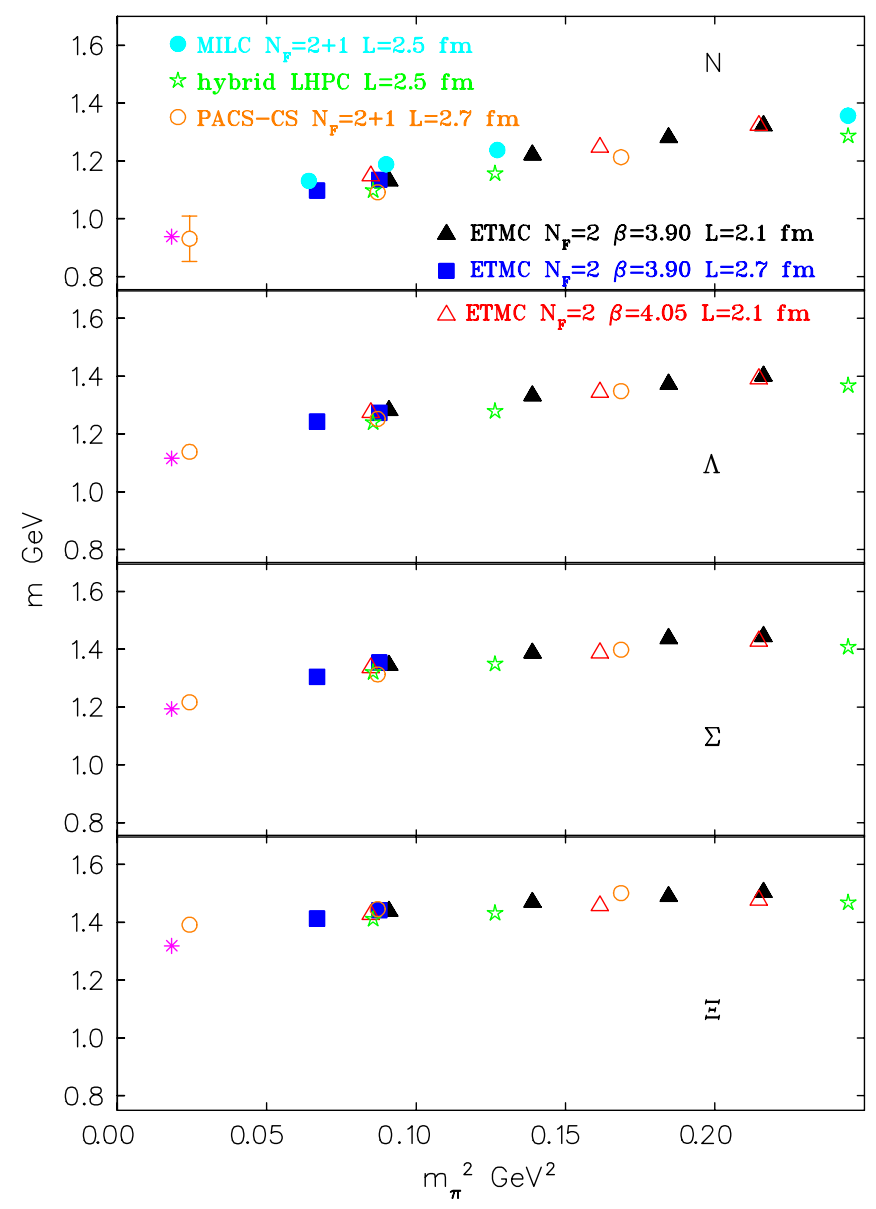

FIG. 22 (color online). Comparison of the masses for the lowlying octet baryons. Results from this work are shown by the filled (black) triangles for $L=2.1 \mathrm{fm}$ and (blue) squares for $L=2.7 \mathrm{fm}$ with $a=0.089 \mathrm{fm}$ and with the open (red) triangles for $L=2.1 \mathrm{fm}$ and $a=0.070 \mathrm{fm}$. Results with the hybrid action (LHPC) are shown with the (green) asterisks for $a=$ $0.124 \mathrm{fm}$ and results using $N_{f}=2+1$ Clover fermions (PACS$\mathrm{CS}$ ) with the open (orange) circles and $a=0.0907 \mathrm{fm}$. For the nucleon we also show results using $N_{f}=2+1$ asqtad improved staggered fermions (MILC) denoted by the filled (light blue) circles. The physical masses are shown by the (purple) star. determined from the nucleon mass to convert physical units. Results from the other collaborations are converted to physical units using the lattice spacing that they provide. The level of agreement of lattice QCD results using a variety of fermion discretization schemes before taking the continuum limit or other lattice artifacts into account is quite impressive. Small discrepancies seen mainly in the decuplet masses can be attributed to lattice artifacts. In particular, results using asqtad improved staggered fermions may suffer the most from discretization errors. The MILC Collaboration has simulations on finer lattices and an update on the masses is expected in the near future. We note that results very close to the physical point obtained

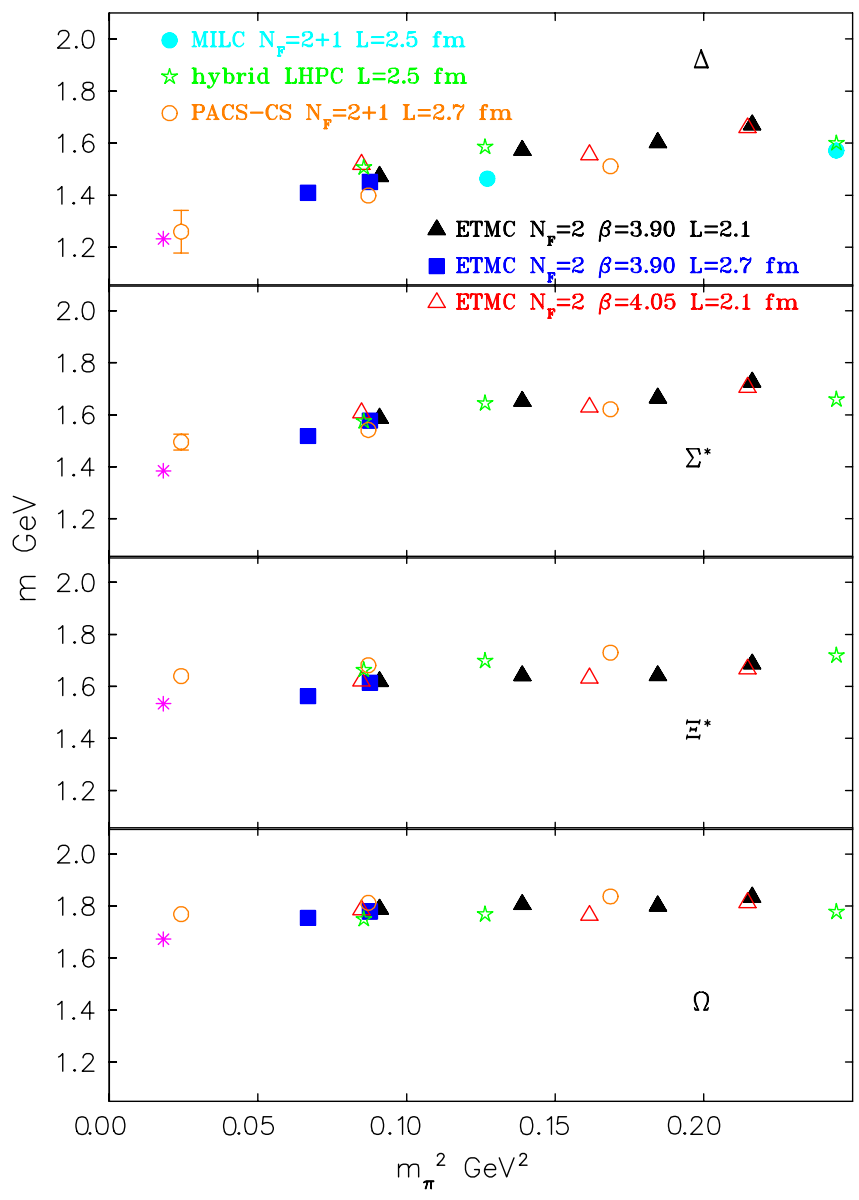

FIG. 23 (color online). Comparison of masses for the lowlying decuplet baryons. The notation is the same as that of Fig. 22. 


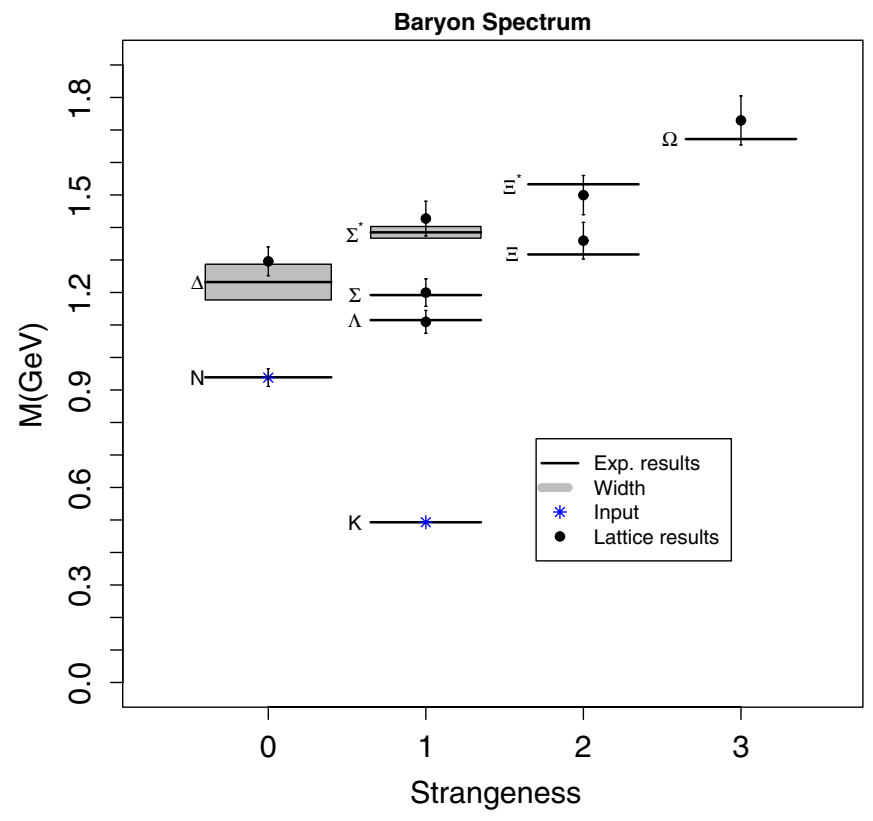

FIG. 24 (color online). The octet and decuplet spectrum. The values shown are obtained after taking the continuum limit and after the extrapolation to the physical point is carried out. For the nucleon and $\Delta$ we used $\mathcal{O}\left(p^{3}\right)$ and for the rest NLO SU(2) $\mathrm{HB} \chi \mathrm{PT}$ for the chiral extrapolations.

using Clover fermions from the PACS-CS Collaboration [6] may have large finite volume effects due to the fact that $m_{\pi} L<3.5$ in this simulation.

Finally we show our continuum results in Fig. 24. We take the continuum limit using results at $\beta=3.9$ and $\beta=4.05$ after interpolating at a given value of $r_{0} m_{\pi}$. The continuum values used are collected in Tables XIII and XIV for the octet and decuplet, respectively. Residual cutoff effects that may result from using Eq. (10) to estimate $\mu_{s}$ at $\beta=4.05$ are not included in the systematic errors. For the nucleon and the $\Delta$ we use $\mathcal{O}\left(p^{3}\right)$ to extrapolate to the physical point as done in our previous work [4]. For the strangeness nonzero baryons we use NLO SU(2) $\mathrm{HB} \chi \mathrm{PT}$ to extrapolate to the physical point. In the statistical error we have added the error arising from the uncertainty in $r_{0}^{N}$, i.e. the difference in the resulting masses when we use $r_{0}^{N}=0.471 \mathrm{fm}$ and $r_{0}^{N} \pm 0.021 \mathrm{fm}$ to set the scale. As can be seen, our results compare well with experiment within the estimated uncertainties.

\section{SUMMARY AND CONCLUSIONS}

The focus of this work is the computation of the lowlying baryon masses using twisted mass fermions at maximal twist. It is in line with ongoing efforts by other lattice collaborations worldwide to predict the baryon spectrum from first principles. Comparison of lattice results with experiment is regarded as an important benchmark for lattice QCD and justifies the use of different lattice actions, each with different systematic errors. For example, the twisted mass action with only one parameter to tune, provides automatic $\mathcal{O}(a)$ improvement. However it restores isospin symmetry only in the continuum limit. We have examined the consequences of isospin breaking on the baryon masses and found them to be either small or compatible with zero. On our finer lattice at $\beta=4.05$ nonvanishing isospin breaking is obtained only in the case of the Cascade where we find that $m_{\Xi^{0}}-m_{\Xi^{-}} \sim$ $50 \mathrm{MeV}$. Finite volume corrections are estimated in the case of the nucleon and found to be smaller than statistical errors. The continuum extrapolation using results at $\beta=$ 3.9 and $\beta=4.05$ are verified using a finer lattice at $\beta=$ 4.2 in the case of the nucleon supporting the analysis carried out. Therefore this study shows that both cutoff effects and finite volume corrections are small and continuum results can be extracted using lattice data at $\beta=3.9$ and $\beta=4.05$.

An investigation of the Gell-Mann-Okubo relations has been carried out. For the octet baryon masses we find that these relations are satisfied at all pion masses even at a nonvanishing lattice spacing. For the decuplet baryon masses we see deviations and it will be interesting to study these relations at finer values of the lattice spacing and smaller quark masses.

Comparison of the results at given lattice spacings with those of other collaborations reveals consistency among groups using improved actions with lattice spacing being smaller than $0.1 \mathrm{fm}$. This is a nontrivial consistency check of several lattice formulations directly on lattice data without the necessity of any extrapolations. This level of agreement among different lattice actions is a welcome outcome of the collective effort of several collaborations. The final continuum results at the physical limit shown in Fig. 24 are in excellent agreement with experiment. The largest uncertainty in the final value comes from systematic errors in setting the scale, which are an order of magnitude larger than statistical errors.

Besides the masses we have extracted from the various fits the $\sigma$ term. To $\mathcal{O}\left(p^{3}\right) \chi \mathrm{PT}$ we find a value of 64(1) $\mathrm{MeV}$ for the nucleon $\sigma$ term. Allowing the coefficient of the cubic term in $m_{\pi}$ to be determined from the data yields a smaller value of 39(12) MeV albeit with a large statistical error. Fitting with the latter Ansatz produces for all baryons expect the $\Omega$ a value of the $\sigma$ term compatible with that of the nucleon. Clearly this is a result that requires further study. In particular, results at smaller pion masses will help to better determine the curvature of fits.

The next step for the ETM Collaboration is to perform the analysis using a dynamical strange quark. Within the twisted mass formalism this is accomplished by simulating a nondegenerate doublet. Such $N_{F}=2+1+1$ simulations are already available at two values of the lattice spacing [56] comparable to the ones studied in this work. This future study will provide a nice comparison to the 
present work and enable us to gauge unquenching effects in the strange quark sector.

\section{ACKNOWLEDGMENTS}

We would like to thank all members of ETMC for the very enjoyable collaboration and the many fruitful discussions that took place during the development of this work. J.C. is grateful to A. Walker-Loud for several constructive discussions on the $\chi \mathrm{PT}$ results. This work was performed using HPC resources from GENCI (IDRIS and CINES) Grant No. 2009-052271. We have also largely benefited from computer and storage resources in the CCIN2P3
(Lyon). Computer time for this project was also made available to us by the John von Neumann-Institute for Computing on the JUMP and Jugene systems at the research center in Jülich and the Stella system at the Donald Smits Center for Information Technology in Groningen. We thank the staff members for their kind and sustained support. This work has been supported in part by the DFG Sonderforschungsbereich/ Transregion SFB/TR9 and by funding received from the Cyprus Research Promotion Foundation under Contracts No. EPYAN/0506/08,

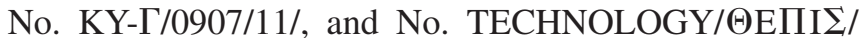
0308(BE)/17.
[1] K. Jansen, arXiv:0810.5634.

[2] C. W. Bernard et al., Phys. Rev. D 64, 054506 (2001).

[3] C. Aubin et al. (MILC Collaboration), Phys. Rev. D 70, 114501 (2004).

[4] C. Alexandrou et al. (European Twisted Mass Collaboration), Phys. Rev. D 78, 014509 (2008).

[5] A. Ali Khan et al. (QCDSF-UKQCD Collaboration), Nucl. Phys. B689, 175 (2004).

[6] S. Aoki et al. (PACS-CS Collaboration), Phys. Rev. D 79, 034503 (2009).

[7] S. Durr et al., Science 322, 1224 (2008).

[8] A. Walker-Loud et al., Phys. Rev. D 79, 054502 (2009).

[9] D. J. Antonio et al. (RBC and UKQCD Collaborations), Proc. Sci., LAT2006 (2006) 189.

[10] D. J. Antonio et al. (RBC and UKQCD Collaborations), Proc. Sci., LAT2006 (2006) 189.

[11] B. Blossier et al. (European Twisted Mass Collaboration), J. High Energy Phys. 04 (2008) 020.

[12] B. Blossier et al., J. High Energy Phys. 07 (2009) 043.

[13] P. Boucaud et al. (ETM Collaboration), Phys. Lett. B 650, 304 (2007).

[14] P. Boucaud et al. (ETM Collaboration), Comput. Phys. Commun. 179, 695 (2008).

[15] R. Frezzotti and G. Rossi, Proc. Sci., LAT2007 (2007) 277.

[16] P. Dimopoulos et al. (ETM Collaboration), arXiv: 0810.2873.

[17] V. Drach et al. (ETM Collaboration), Proc. Sci., LAT2008 (2008) 123.

[18] P. Weisz, Nucl. Phys. B212, 1 (1983).

[19] P. Boucaud et al., Comput. Phys. Commun. 179, 695 (2008).

[20] R. Frezzotti, G. Martinelli, M. Papinutto, and G. Rossi, J. High Energy Phys. 04 (2006) 038.

[21] R. Frezzotti and G. Rossi, J. High Energy Phys. 08 (2004) 007.

[22] K. Osterwalder and E. Seiler, Ann. Phys. (N.Y.) 110, 440 (1978).

[23] R. Frezzotti and G. C. Rossi, J. High Energy Phys. 10 (2004) 070.
[24] A. M. Abdel-Rehim, R. Lewis, R. M. Woloshyn, and J. M. S. Wu, Eur. Phys. J. A 31, 773 (2007).

[25] A. M. Abdel-Rehim, R. Lewis, R. M. Woloshyn, and J. M. S. Wu, Phys. Rev. D 74, 014507 (2006).

[26] C. Urbach, Proc. Sci., LAT2007 (2007) 022.

[27] V. Lubicz and C. Tarantino (private communication).

[28] P. Dimopoulos, R. Frezzotti, G. Herdoiza, C. Urbach, and U. Wenger (ETM Collaboration), Proc. Sci., LAT2007 (2007) 102 [arXiv:0710.2498].

[29] G. Martinelli, C. Pittori, C. T. Sachradja, M. Testa, and A. Vladikas, Nucl. Phys. B445, 81 (1995).

[30] C. Alexandrou, M. Constantinou, and T. Korzec (private communication); (unpublished).

[31] B. L. Ioffe, Nucl. Phys. B188, 317 (1981).

[32] D. B. Leinweber, R. M. Woloshyn, and T. Draper, Phys. Rev. D 43, 1659 (1991).

[33] D. B. Leinweber, T. Draper, and R. M. Woloshyn, Phys. Rev. D 46, 3067 (1992).

[34] M. Albanese et al. (APE Collaboration) Phys. Lett. B 192, 163 (1987).

[35] U. Wolff (ALPHA Collaboration), Comput. Phys. Commun. 156, 143 (2004).

[36] J.F. Donoghue, E. Golowich, and B. R. Holstein, Cambridge Monogr. Part. Phys., Nucl. Phys., Cosmol. 2, 1 (1992)

[37] S. R. Beane, K. Orginos, and M. J. Savage, Phys. Lett. B 654, 20 (2007).

[38] M. Procura, T. R. Hemmert, and W. Weise, Phys. Rev. D 69, 034505 (2004).

[39] S. Steininger, U.-G. Meissner, and N. Fettes, J. High Energy Phys. 09 (1998) 008.

[40] V. Bernard, T. Hemmert, and U.-G. Meissner, Nucl. Phys. A732, 149 (2004).

[41] V. Bernard, T. Hemmert, and U.-G. Meissner, Phys. Lett. B 622, 141 (2005).

[42] N. Fettes, U.-G. Meissner, and S. Steininger, Nucl. Phys. A640, 199 (1998).

[43] M. Procura et al., Phys. Rev. D 73, 114510 (2006).

[44] D. R. Entem and R. Machleidt, Phys. Rev. C 66, 014002 (2002).

[45] E. Epelbaum, W. Glockle, and U.-G. Meissner, Nucl. 
Phys. A747, 362 (2005).

[46] K. I. Ishikawa et al. (PACS-CS Collaboration), Phys. Rev. D 80, 054502 (2009).

[47] E. Collaboration (to be published).

[48] C. Alexandrou et al. (ETM Collaboration), Proc. Sci., LAT2007 (2007) 087.

[49] B. C. Tiburzi and A. Walker-Loud, Phys. Lett. B 669, 246 (2008).

[50] M. Frink, U.-G. Meissner, and I. Scheller, Eur. Phys. J. A 24, 395 (2005).

[51] M. Frink and U.-G. Meissner, J. High Energy Phys. 07
(2004) 028.

[52] J. Gasser, M. Sainio, and A. Svarc, Nucl. Phys. B307, 779 (1988).

[53] M. M. Nagels et al., Nucl. Phys. B147, 189 (1979).

[54] M. M. Nagels, T. A. Rijken, and J. J. de Swart, Phys. Rev. D 20, 1633 (1979).

[55] B. C. Tiburzi and A. Walker-Loud, Nucl. Phys. A764, 274 (2006).

[56] R. Baron et al. (ETM Collaboration), Proc. Sci., LATTICE2008 (2008) 094. 